\title{
Dynamical Insights into Extreme Short-Term Precipitation Associated with Supercells and Mesovortices $\mathscr{O}$
}

\author{
ERIK R. NIELSEN AND RUSS S. SCHUMACHER \\ Department of Atmospheric Science, Colorado State University, Fort Collins, Colorado
}

(Manuscript received 15 December 2017, in final form 23 May 2018)

\begin{abstract}
In some prominent extreme precipitation and flash flood events, radar and rain gauge observations have suggested that the heaviest short-term rainfall accumulations (up to $177 \mathrm{~mm} \mathrm{~h}^{-1}$ ) were associated with supercells or mesovortices embedded within larger convective systems. In this research, we aim to identify the influence that rotation has on the storm-scale processes associated with heavy precipitation. Numerical model simulations conducted herein were inspired by a rainfall event that occurred in central Texas in October 2015 where the most extreme rainfall accumulations were collocated with meso- $\beta$-scale vortices. Five total simulations were performed to test the sensitivity of precipitation processes to rotation. A control simulation, based on a wind profile from the aforementioned event, was compared with two experiments with successively weaker low-level shear. With greater environmental low-level shear, more precipitation fell, in both a point-maximum and an area-averaged sense. Intense, rotationally induced low-level vertical accelerations associated with the dynamic nonlinear perturbation vertical pressure gradient force were found to enhance the low- to midlevel updraft strength and total vertical mass flux and allowed access to otherwise inhibited sources of moisture and CAPE in the higher-shear simulations. The dynamical accelerations, which increased with the intensity of the low-level shear, dominated over buoyant accelerations in the low levels and were responsible for inducing more intense low-level updrafts that were sustained despite a stable boundary layer.
\end{abstract}

\section{Introduction}

Throughout the United States, flash flooding continues to threaten life and property despite increased awareness and forecasting advances (e.g., Ashley and Ashley 2008). Forecasting the extreme rainfall and often associated flash flooding presents many challenges because one must correctly predict both the occurrence and magnitude of extreme rainfall to correctly predict the occurrence and magnitude (i.e., potential impacts) of the flash flooding (e.g., Doswell et al. 1996). The accurate numerical prediction $^{1}$ and nowcasting ${ }^{2}$ of rainfall accumulations

\footnotetext{
${ }^{1}$ This is known as quantitative precipitation forecasting (QPF).

${ }^{2}$ This is known as quantitative precipitation estimation (QPE), which is the real-time estimation of rainfall accumulations using rain gauge observations combined with remote sensing techniques (e.g., Zhang et al. 2016).
}

Supplemental information related to this paper is available at the Journals Online website: https://doi.org/10.1175/JAS-D-170385.s1.

Corresponding author: Erik Nielsen, erik.nielsen@colostate.edu remain a continued challenge in the meteorological community (e.g., Fritsch and Carbone 2004; Novak et al. 2011; Zhang et al. 2016; Herman and Schumacher 2018).

At the most basic level, extreme precipitation accumulations over some area is required before flash flooding can occur. For large rainfall accumulations to occur, high rain rates must persist in a location for a long period of time (e.g., Chappell 1986; Doswell 1994; Doswell et al. 1996). From Doswell et al. (1996), the total precipitation at a location can be expressed simply as

$$
P=\bar{R} D,
$$

where $\bar{R}$ is the average rainfall rate and $D$ is the rainfall duration. The average rainfall rate $\bar{R}$ is often not particularly illustrative of the ingredients needed for extreme rainfall. However, the instantaneous rainfall rate $R$ can be broken down into separate illustrative elements; $R$ can be expressed as

$$
R=E w q,
$$

where $E$ is the precipitation efficiency, $q$ is the water vapor mixing ratio of the rising air, and $w$ is the ascent rate. The precipitation efficiency $E$ is a proportionality 
constant relating rainfall rate to water vapor flux [see appendix of Doswell et al. (1996)]. As shown by Eq. (1), high precipitation accumulations could also be the result of slow-moving convective systems or "echo training" [i.e., maximizing the duration $D$ in Eq. (1)]. Quasistationary or back-building mesoscale convective systems such as these can be especially prevalent flash flood producers (e.g., Bluestein and Jain 1985; Chappell 1986; Doswell et al. 1996; Schumacher and Johnson 2005); however, the foremost focus of this research will not be on storm motion or propagation but rather on dynamical accelerations, specifically related to the presence of rotation, within the storms that could lead to high rainfall rates. Doswell et al. (1996) theorized that intense nonbuoyant accelerations, which are a substantial source for positive vertical momentum in supercells (e.g., Rotunno and Klemp 1982; Weisman and Klemp 1984), and the resulting intense updrafts [i.e., $w$ in Eq. (2)] create an increased potential for intense rainfall rates that is otherwise lessened (all else being equal) without the presence of rotation.

Within the general classifications of extreme-rainfallproducing storms, it has often been observed that precipitation is associated with mesoscale vortices on various scales, including supercells (e.g., Moller et al. 1994; Smith et al. 2001; Hitchens and Brooks 2013; Schumacher 2015a) and larger mesoscale structures, such as mesoscale convective vortices (MCVs; e.g., Bosart and Sanders 1981; Fritsch et al. 1994; Trier et al. 2000a; Schumacher and Johnson 2009; Morales et al. 2015; Nielsen et al. 2016b). Further, from a broader impacts point of view, the presence of tornadoes (i.e., from rotation) and flash flooding (i.e., from extreme rainfall) in the same place at the same time presents a dangerous situation where lifesaving actions are contradictory [tornado and flash flood events (TORFFs); Nielsen et al. 2015, 2016a; Bunkers and Doswell 2016]. Additionally, tropical cyclones can possess environmental characteristics conducive for the development of mesoscale rotation and supercells, especially within attendant rainbands (i.e., high low-level shear; McCaul and Weisman 2001; Baker et al. 2009; Morin and Parker 2011; Edwards et al. 2012; Wang et al. 2015). The presence of rotation and flooding in these high-impact weather events further motivates the need to investigate any possible relationship between the dynamics of rotation and extreme rainfall production.

Supercells were once thought not to produce extreme rainfall/rain rates, because of low precipitation efficiency, $E$ in Eq. (2), associated with large values of convective available potential energy (CAPE), shear, and hail production (e.g., Marwitz 1972; Foote and Fankhauser 1973; Browning 1977). However, recent studies (Smith et al. 2001; Duda and Gallus 2010; Hitchens and Brooks 2013) have shown that supercells have been responsible for extreme rainfall events, even noted world record accumulations (Dalrymple 1937), and might be underrepresented causes of flash floods. A specific subclass of supercells, known as high-precipitation (HP) supercells (e.g., Moller et al. 1994), are known to produce more precipitation than other supercell storms. HP supercells produce the greatest threat of flash flooding, among all the supercell archetypes, with multiple flash flood events caused by such storms (e.g., Moller et al. 1994; Smith et al. 2001; Bunkers and Doswell 2016). The high rain rates in HP supercells has been attributed to the ability of an intense and/or spatially large updraft to ingest significant amounts of water vapor (e.g., Smith et al. 2001; Beatty et al. 2008), which fits into the rotational enhancement framework discussed in Doswell et al. (1996).

Specifically, the observed extreme rain rates seen in supercells, despite low precipitation efficiency, can possibly be explained by noting that supercells have an additional positive source of vertical momentum [i.e., $w$ in Eq. (2)] from the nonlinear dynamic vertical perturbation pressure gradient force associated with the mesocyclone (e.g., Weisman and Klemp 1984; Doswell et al. 1996). The illustration of the effects that rotation has on vertical pressure gradients can be found in the mathematical decomposition of the vertical perturbation pressure $p^{\prime}$ gradient force (VPPGF) into buoyant (i.e., $p_{B}^{\prime}$ ), dynamic linear (i.e., $p_{\mathrm{DL}}^{\prime}$ ), and dynamic nonlinear (i.e., $p_{\mathrm{DNL}}^{\prime}$ ) components (e.g., Rotunno and Klemp 1982; Klemp 1987; Markowski and Richardson 2010). For the sake of brevity, the full decomposition will not be undertaken here. However, the resulting expanded vertical momentum equation following this decomposition, excluding the Coriolis force, can be expressed as

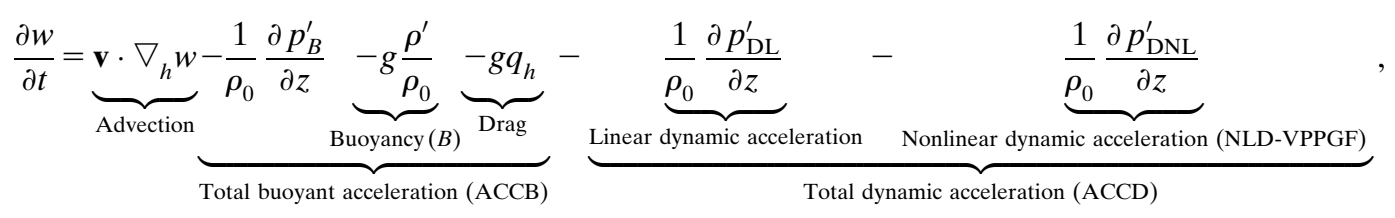


where the total buoyant acceleration (ACCB) is the acceleration that results from the combined effects of thermodynamically driven buoyancy, hydrometeor drag (i.e., $g q_{h}$, where $q_{h}$ is the total hydrometeor mixing ratio), and the vertical gradient in the buoyancy pressure field. The total dynamic acceleration (ACCD) is associated with the effects of both the linear and nonlinear dynamic (NLD-VPPGF) perturbation pressure-induced accelerations. To conceptualize what physical processes affect the individual terms of the VPPGF (i.e., $p_{\mathrm{DL}}^{\prime}, p_{\mathrm{DNL}}^{\prime}$, and $\left.p_{B}^{\prime}\right)$, a simplified, approximate decomposition of the perturbation pressure $p^{\prime}$ can be written, following Markowski and Richardson (2010), for well-behaved, incompressible, storm-scale flows as

$$
p^{\prime} \propto \underbrace{\underbrace{e_{i j}^{2}}_{\text {Splat }}-\underbrace{\frac{1}{2}\left|\boldsymbol{\omega}^{\prime}\right|^{2}}_{\text {Spin }}}_{\text {Nonlinear dynamic }\left(p_{\mathrm{DNL}}^{\prime}\right)}+\underbrace{2 \mathbf{S} \cdot \nabla_{h} w^{\prime}}_{\text {Linear dynamic }\left(p_{\mathrm{DL}}^{\prime}\right)}-\underbrace{\frac{\partial B}{\partial z}}_{\text {Buoyant }\left(p_{B}^{\prime}\right)},
$$

where $e_{i j}$ is the deformation tensor, $\boldsymbol{\omega}$ is the total vorticity of the perturbation wind, $B$ is buoyancy, $w$ is vertical motion, and $\mathbf{S}$ is the mean environmental wind shear vector. The nonlinear dynamic pressure perturbation (i.e., $p_{\mathrm{DNL}}^{\prime}$ ) is made up of the "splat" and "spin" terms, which produce opposite-signed pressure perturbations [Eq. (4)]. The spin term implies that strong rotation around any axis in any direction is associated with a negative pressure perturbation. However, rotation around a vertical axis will be the focus of the research presented here. This negative pressure perturbation can act to dynamically enhance or retard the strength of the updraft (i.e., $w$ ) depending on the vertical distribution of the rotation. ${ }^{3}$ The influence of rotation and the induced pressure perturbation highlights the physical mechanism by which rotation could potentially enhance rain rates. Although the influence of the VPPGF has been investigated in regards to supercells and tornadogenesis, little attention has been devoted to its impact on precipitation processes when supercells or embedded mesovortices are present.

On the convective scale, cells that produce the most extreme rain rates have been shown to be associated with a positive potential vorticity (PV) monopole, compared to the expected PV dipole that is seen in other convective storms (i.e., the positive PV anomaly dominates over the negative anomaly; Chagnon and Gray 2009;

\footnotetext{
${ }^{3}$ For more discussion on this, especially the latter point, see work on mesovortices embedded in squall lines by Weisman and Trapp (2003) and Trapp and Weisman (2003).
}

Weijenborg et al. 2015, 2017), which can persist even after the storm decays. This implies that the convective cells that produce the most intense rain rates have supercellular-like structure (i.e., a long-lived, rotating updraft) in PV space (Weijenborg et al. 2017). The positive PV monopole structure described here is, similar to what is known about $\mathrm{MCV}^{4}$ development (Haynes and McIntyre 1987; Raymond and Jiang 1990; Hertenstein and Schubert 1991; Trier et al. 2000b), partially influenced by the latent heat release in a convective storm's updraft and further illustrates the pathway for positive feedbacks to exists between rotation and intense precipitation (e.g., Schumacher et al. 2013; Morales et al. 2015; Nielsen and Schumacher 2016).

The positive or negative effects on a storm's updraft associated with the NLD-VPPGF can alter the depth of the layer(s) that serves as the primary energy source for buoyant ascent in updrafts. In the United States, the majority of warm-season heavy rainfall flash flood events are the result of MCSs (e.g., Fritsch et al. 1986; Schumacher and Johnson 2006) and tend to occur overnight (e.g., Stevenson and Schumacher 2014; Herman and Schumacher 2016). The latter point implies the presence of a stable nocturnal planetary boundary layer (PBL), and the presence of a nocturnal low-level jet (e.g., Bonner 1968), which is an important synoptic- to mesoscale feature common to warm-season MCSs (e.g., Parker and Johnson 2000; Moore et al. 2003; Schumacher and Johnson 2005) that can serve to enhance $0-1-\mathrm{km}$ shear. Surface-to-1-km shear, specifically, has been found to be particularly favorable for tornado (e.g., Craven et al. 2004) and mesovortex development (e.g., Weisman and Trapp 2003; Trapp and Weisman 2003; Atkins and St. Laurent 2009). The shear is associated with environmental horizontal vorticity confined to the low levels that, through its tilting and ingestion into a developing updraft, effectively lowers the base of and strengthens the midlevel mesocyclone (e.g., Markowski et al. 2012; Markowski and Richardson 2014; Coffer and Parker 2015), because of the development of rotation around a vertical axis and the dynamical enhancement (i.e., from the NLD-VPPGF) of the updraft. This lowering, in turn, makes it easier for the rotationally induced NLD-VPPGF to lift negatively buoyant air, especially in the case of weak cold pools, in the PBL (e.g., Nowotarski et al. 2011; Davenport and Parker 2015) that can be an additional source of moisture and instability to the storm (Schumacher 2015b). Further,

\footnotetext{
${ }^{4}$ It should be noted that MCVs themselves do not often possess large rotation rates compared to mesovortices or supercell mesocyclones (e.g., James and Johnson 2010) and therefore have limited sources of vertical momentum from the VPPGF.
} 

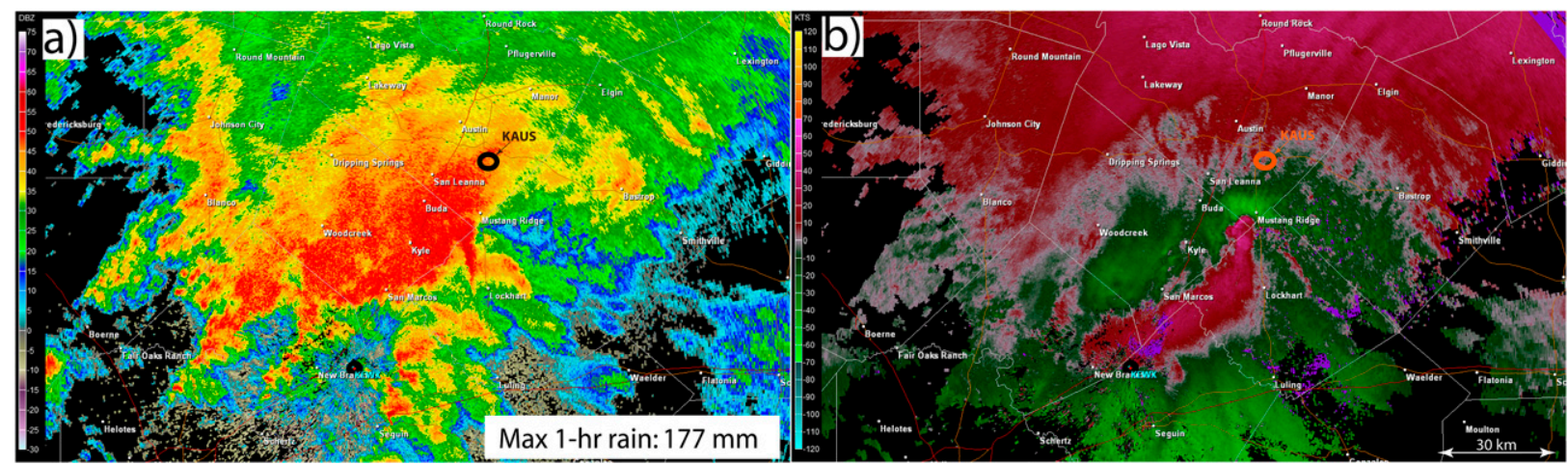

FIG. 1. (a) Radar reflectivity and (b) base velocity for the case of interest where intense rainfall accumulations were observed attendant with mesoscale rotation 1342 UTC 30 Oct 2015 from Austin/San Antonio (KEWX) radar. The METAR station where rainfall accumulations were observed is labeled on the radar reflectivity plot. Maximum 1-h rainfall observation from local METAR or mesonet networks are labeled for the case.

this process can also serve to create a positive feedback between horizontal environmental vorticity, rotation rate, updraft strength, and magnitude of the NLD-VPPGF (Coffer and Parker 2015).

With these potential interactions in mind, it is hypothesized that the presence of moist convective meso$\gamma$ - to meso- $\beta$-scale vortices associated with intense $0-1-\mathrm{km}$ shear have an increased propensity to produce extreme rain rates, all else being equal, compared to other storm types. This is accomplished by, first, dynamically enhancing the updraft through the nonlinear dynamic component of the vertical pressure perturbation gradient acceleration and, second, with this enhancement, dynamically lifting otherwise inhibited parcels that still possess moisture and instability from an otherwise stable boundary layer. Furthermore, if the presence of mesoscale rotation can serve to enhance rain rates, it perhaps could serve as a compounding physical explanation, in addition to echo training, for the occurrence and frequency of TORFFs in the United States (Nielsen et al. 2015). In this study, numerical modeling experiments where the $0-1-\mathrm{km}$ shear ${ }^{5}$ is varied are used to explore the dynamical effects of rotation on precipitation processes. The resulting storm characteristics, precipitation accumulations, and induced mesoscale dynamic accelerations will be examined. Section 2 provides a description of the extreme rainfall event used as the basis for the model initial conditions, section 3 describes the methodology used in this study, section 4 presents the results of simulations with planetary rotation, section 5 presents the results with planetary rotation

\footnotetext{
${ }^{5}$ From this point on in this manuscript, the "wind shear" verbiage will refer to the bulk wind difference over the specified layer, and the units will reflect as such.
}

included, and section 6 summarizes the results and presents a discussion about the conclusions.

\section{Case of interest}

Although quantifying the proportion of extreme shortterm rain events associated with low-level rotation is beyond the scope of this study and is a topic of ongoing research by the authors, one example that served as the initial motivation for this study is summarized here.

A TORFF that occurred in south-central Texas on 30 October 2015 (Figs. 1a,b) will serve as the basis for the numerical modeling experiments presented in this study. A very strong, long-lived mesoscale vortex developed northeast of San Antonio, Texas, within an already developed MCS near 1200 UTC that day. As the vortex moved north toward Austin, Texas, over the next $3 \mathrm{~h}$ (Fig. 1b), hourly rainfall observations of $100-177 \mathrm{~mm}$ were observed by several Lower Colorado River Authority (LCRA) gauges along its path (not shown; LCRA 2017). Furthermore, an hourly accumulation of $146.3 \mathrm{~mm}$ was recorded at Austin-Bergstrom International Airport (KAUS; Figs. 1a,b). A total of 11 flash flood and 11 tornado warnings were issued by NWS Austin/San Antonio during the event, with a total of four tornadoes, including two rated EF2 on the enhanced Fujita scale, surveyed, and five fatalities associated with the flash flooding (NCEI 2017).

South-central Texas was positioned in the southern portion of a subtropical jet streak (Fig. 2a) downstream of an approaching long-wave trough by 0900 UTC 30 October 2015 (Fig. 2a). The convection that eventually formed into the MCS in question initiated over the Mexican Plateau and Texas-Mexico border near 0600 UTC that day, as the upper-level forcing moved into the area. Significant southeasterly flow off the Gulf 

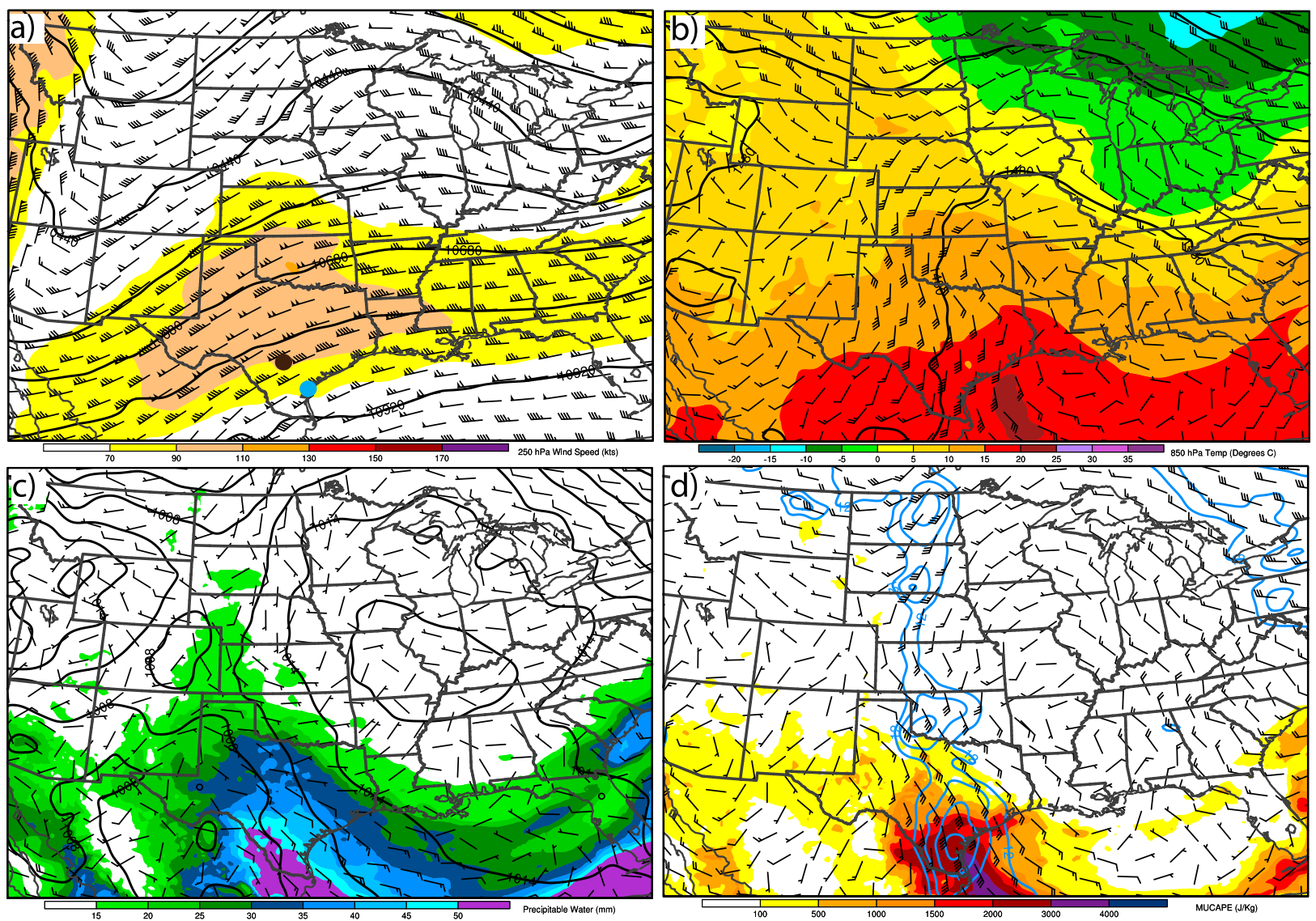

FIG. 2. RAP (Benjamin et al. 2016) analyses at 0900 UTC 30 Oct 2015. (a) The 250-hPa isotachs (shaded every $20 \mathrm{kt}$ over $70 \mathrm{kt}$ ), 250 -hPa geopotential height (contoured every $120 \mathrm{~m}$ ), and $250-\mathrm{hPa}$ wind barbs (half barb $=5 \mathrm{kt}$, full barb $=10 \mathrm{kt}$, pennant $=50 \mathrm{kt}$ ); cyan dot represents the approximate location of the Corpus Christi sounding in Fig. 3a, and black dot represents the location of San Antonio. (b) The 850-hPa geopotential height (contoured every $25 \mathrm{~m}$ ), 850-hPa wind barbs, and 850 -hPa temperature (shaded every $5^{\circ} \mathrm{C}$ from $-20^{\circ}$ to $35^{\circ} \mathrm{C}$ ). (c) PWAT (shaded contours every $5 \mathrm{~mm}$ for values from 10 to $50 \mathrm{~mm}$ ), $10 \mathrm{~m}$ wind barbs, and mean sea level pressure (MSLP; contoured every $3 \mathrm{hPa}$ ). (d) Most unstable CAPE (MUCAPE; shaded at $100 \mathrm{~J} \mathrm{~kg}^{-1}$ then every $500 \mathrm{~J} \mathrm{~kg}^{-1}$ above $500 \mathrm{~J} \mathrm{~kg}^{-1}$ ), $900-\mathrm{hPa}$ wind barbs, and 900-hPa isotachs (contoured every $3 \mathrm{~m} \mathrm{~s}^{-1}$ above $12 \mathrm{~m}^{-1}$ ).

of Mexico ahead of the upper-level trough provided a reservoir of moisture and buoyancy into the region (Figs. 2c,d), as well as continued warm-air advection (Fig. 2b). The intense low-level flow [i.e., approaching $50 \mathrm{kt}\left(1 \mathrm{kt}=0.51 \mathrm{~m} \mathrm{~s}^{-1}\right)$ at 850 and $900 \mathrm{hPa}$; Figs. 2b,d] also created a strongly sheared low-level environment. The upstream 0000 UTC sounding from Corpus Christi, Texas (KCRP), contained $16.8 \mathrm{~m} \mathrm{~s}^{-1}$ of $0-1-\mathrm{km}$ shear and $52.4 \mathrm{~mm}$ of precipitable water (PWAT) with the surface parcel being slightly inhibited (Fig. 3a). A sounding taken from the 0000 UTC initialization of the Colorado State University (CSU) Advanced Research version of WRF (WRF-ARW; Klemp et al. 2007; Skamarock et al. 2008; Skamarock and Klemp 2008)

\footnotetext{
${ }^{6}$ See Schumacher (2015a) and Peters et al. (2017) for model setup information.
}

numerical model ${ }^{6}$ shows at 1500 UTC a similar low-level kinematic picture with $17.2 \mathrm{~m} \mathrm{~s}^{-1}$ of $0-1-\mathrm{km}$ shear, strong veering in the low-level hodograph, and similar amounts of PWAT (cf. Figs. 3a and 3b). A kinematic profile partially based upon this CSU-WRF Model sounding (Fig. 3b) was used to set up the initial conditions for the experiments described below, because of the sounding's close proximity (both temporally and spatially) to the modeled mesoscale vortex and the lowlevel kinematic similarities to the observed upstream sounding from Corpus Christi.

\section{Methods}

Three-dimensional numerical model simulations were conducted in Cloud Model 1, version 1.18 (CM1; Bryan and Fritsch 2002) in a similar configuration as that described in Schumacher $(2009,2015 b)$. Some of 

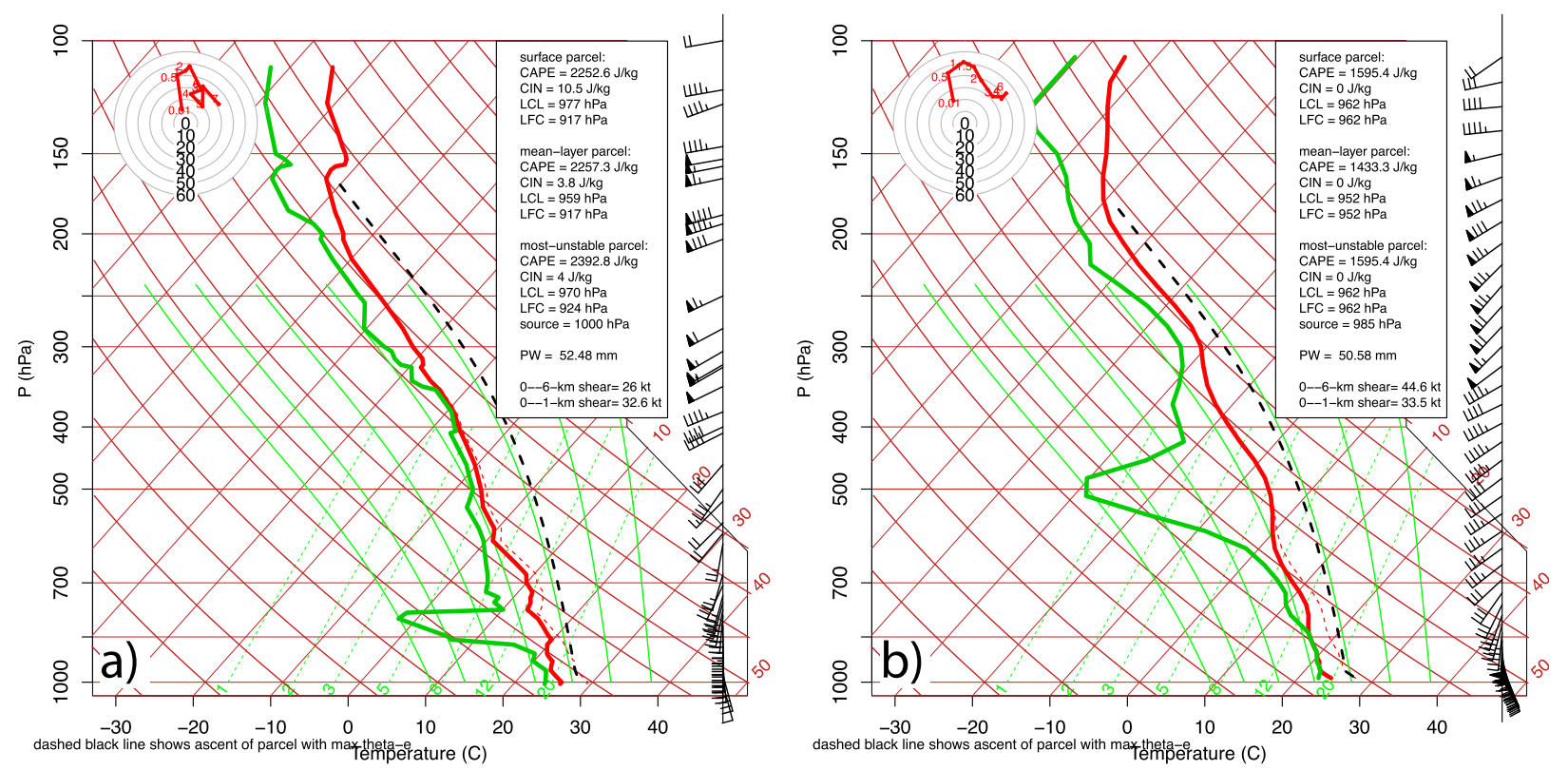

FIG. 3. (a) Observed sounding valid 1200 UTC 30 Oct 2015 from KCRP. (b) Model sounding from the CSU-WRF [see Schumacher (2015a) and Peters et al. (2017) for model setup information] for San Antonio (location denoted by black dot in Fig. 2) valid 1500 UTC 30 Oct 2015. Black dashed line in both soundings represents the temperature of a lifted parcel with the maximum equivalent potential temperature $\theta_{e}$ using the virtual temperature correction. Red dotted line in both soundings represents the virtual temperature correction to the temperature profile.

the more pertinent model specifics include 500-m horizontal grid spacing on a $1200 \times 1200$ gridpoint domain (i.e., $600 \mathrm{~km} \times 600 \mathrm{~km}$ ), a stretched vertical grid with 61 levels, $100-\mathrm{m}$ vertical resolution near the surface, 500-m vertical resolution aloft, free-slip upper and lower boundaries, and open-radiative boundary conditions (Durran and Klemp 1983) that are restricted so the outward mass flux does not exceed the inward. Additionally, radiative processes were excluded, and the Morrison two-moment microphysics scheme with graupel prescribed as the large ice category was used (Morrison et al. 2009). The model domain was translated at a speed of $u=6.5 \mathrm{~m} \mathrm{~s}^{-1}$ and $v=8.0 \mathrm{~m} \mathrm{~s}^{-1}$. As in Schumacher $(2009,2015 b)$, the convection was initiated using a momentum forcing that develops a three-dimensional circular convergence field, which imitates the gradual mesoscale ascent that is typically associated with an MCV, following the methods developed by Loftus et al. (2008). The forcing was horizontally centered at gridpoint $460 \times 460$ (i.e., $230 \mathrm{~km}$ into the domain in both the $x$ and $y$ directions) and vertically centered at $1.4 \mathrm{~km}$, and it had a vertical radius of $1 \mathrm{~km}$ and a horizontal radius of $140 \mathrm{~km}$. The maximum divergence prescribed was $-1 \times 10^{-5} \mathrm{~s}^{-1}$ and increased incrementally over the first $2-3 \mathrm{~h}$, where it levels off at approximately the chosen maximum divergence (convergence).
The initial environmental horizontally homogeneous thermodynamic base-state profile for the simulations undertaken in this study was taken from the composite sounding (Fig. 4a) created by Schumacher and Johnson (2009) over six extreme rainfall events where the lowest kilometer reflects the effects of nocturnal stabilization. The profile is characterized by moist low levels, $50 \mathrm{~mm}$ ( $\sim 2$ in.) of PWAT, moderate CAPE, and no convective inhibition (CIN) for the most unstable parcel sourced at $875 \mathrm{hPa}$. However, nonnegligible CIN $\left(61 \mathrm{~J} \mathrm{~kg}^{-1}\right)$ is present for surface-based parcels. This initial thermodynamic profile was chosen for these simulations because it is smooth relative to the individual cases and excludes the possible influence of noisy case-dependent variations in temperature and moisture from unique cases [i.e., see construction of profile in Schumacher and Johnson (2009)]. Further, passive tracers were placed in the PBL, throughout the entire layer below $750 \mathrm{~m}$, to test whether parcels were ingested from the stable boundary layer present in the initial conditions (Fig. 4a).

The initial wind profile was taken from the aforementioned CSU-WRF Model sounding (i.e., Fig. 3b) valid at 1500 UTC 30 October 2015 near San Antonio. To focus the experiments on the role of low-level wind shear, the shear above $6 \mathrm{~km}$ was removed from the CSU-WRF sounding (Fig. 4b). The resultant wind profile (Fig. 4b) and hodograph (Fig. 4c) represent the 

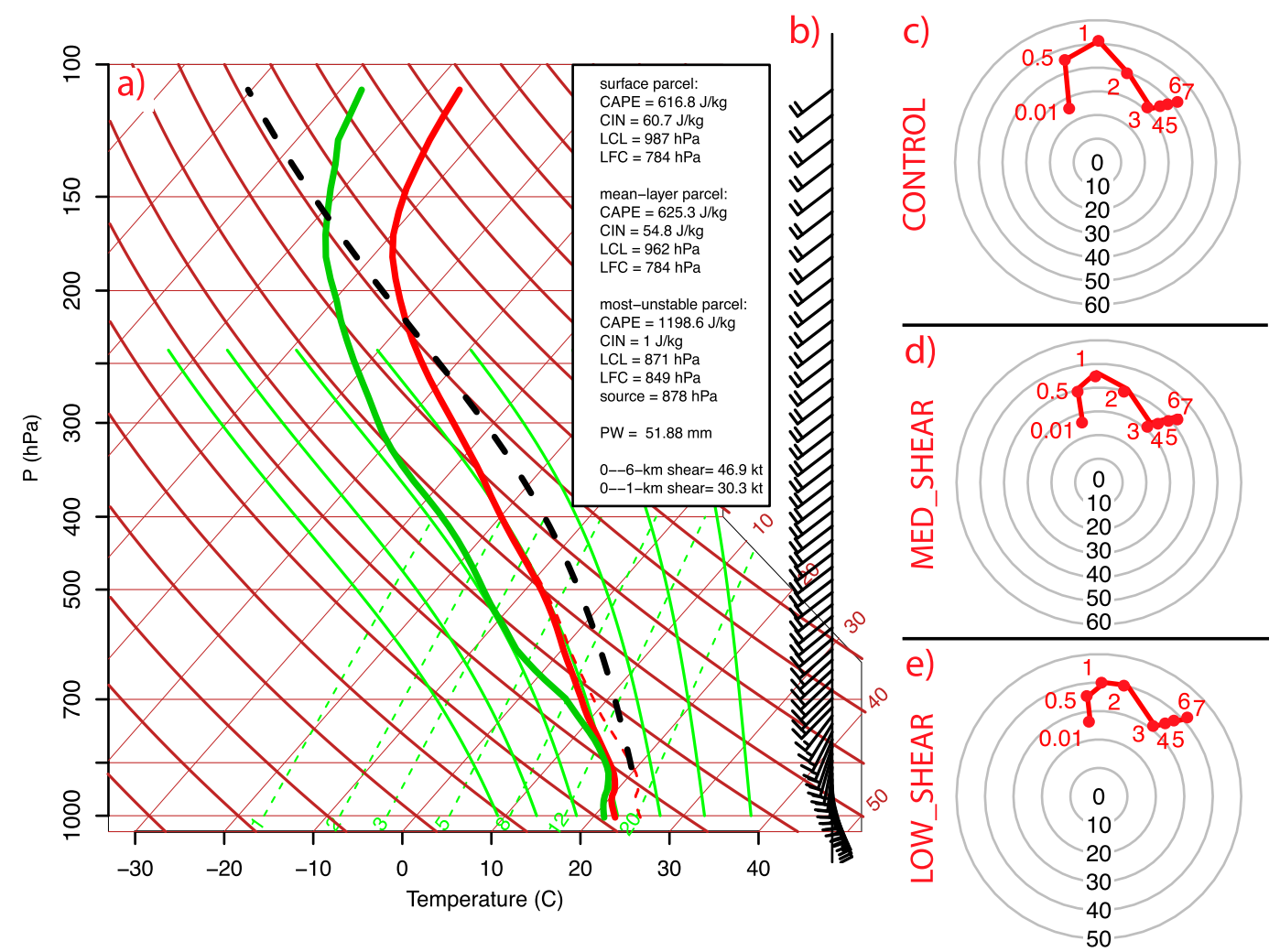

FIG. 4. (a) Composite thermodynamic profile and parcel characteristics for extreme rainfall events from Schumacher and Johnson (2009); red line shows the environmental virtual temperature curve, and black dashed line shows the virtual temperature of a lifted parcel that contains the highest $\theta_{e}$. Hodographs $(\mathrm{kt})$ of wind profile used for wind 0-1-km shear sensitivity experiments for (c) CONTROL, (d) MED_SHEAR, and (e) LOW_SHEAR cases derived from CSU-WRF Model sounding valid 1500 UTC 30 Oct 2015 near San Antonio (e.g., Fig. 3b), where each numeric value along a hodograph trace represents wind vector height $(\mathrm{km})$ at corresponding marker (note LOW_SHEAR hodograph ring maximum is $50 \mathrm{kt}$ compared to $60 \mathrm{kt}$ for other two cases). (b) Wind profile corresponding to $(\mathrm{c})$.

kinematic profile that was used as the control for the quasiidealized experiments presented in this study. The influence of the rotation on precipitation processes was examined by producing a set of simulations in which the low-level wind shear was modified. The primary purpose of these experiments is to explore how changes in the magnitude of the low-level wind shear affects storm dynamics and precipitation production. Thus, we developed two additional wind profiles with weaker lowlevel shear but a similar hodograph shape. Furthermore, these wind profiles were modified slightly so that the predicted motion of a right-moving supercell (using the method of Bunkers et al. 2000) would be the same for all of the simulations. The wind profile in the first run, referred to as CONTROL, was the slightly modified wind profile described above that contains the highest $0-1-\mathrm{km}$ shear of the simulations with a value over $15 \mathrm{~m} \mathrm{~s}^{-1}$ (Fig. 4c and Table 1). The $0-1-\mathrm{km}$ shear was then reduced to approximately 10 and $7.5 \mathrm{~m} \mathrm{~s}^{-1}$ for the medium-shear (Fig. 4d and Table 1; referred to as

TABLE 1. Characteristics of the three wind profiles used in the CONTROL, MED_SHEAR, and LOW_SHEAR experiments. The storm-relative helicity (SRH) is calculated for the Bunkers predicted right-mover storm motion (e.g., Bunkers et al. 2000) of $u=7.3 \mathrm{~m} \mathrm{~s}{ }^{-1}$ and $v=8.3 \mathrm{~m} \mathrm{~s}^{-1}$ (or $220^{\circ}$ at $11 \mathrm{~m} \mathrm{~s}^{-1}$ ) that is approximately equal for each wind profile.

\begin{tabular}{lccc}
\hline \hline & CONTROL & MED_SHEAR & LOW_SHEAR \\
\hline $0-1-\mathrm{km}$ bulk wind difference $\left(\mathrm{m} \mathrm{s}^{-1}\right)$ & 15.2 & 10.7 & 7.6 \\
$0-6-\mathrm{km}$ bulk wind difference $\left(\mathrm{m} \mathrm{s}^{-1}\right)$ & 24.1 & 21.1 & 18.0 \\
$0-1-\mathrm{km} \mathrm{SRH}\left(\mathrm{m}^{2} \mathrm{~s}^{-2}\right)$ & 286 & 161 & 96 \\
$0-3-\mathrm{km} \mathrm{SRH}\left(\mathrm{m}^{2} \mathrm{~s}^{-2}\right)$ & 406 & 281 & 184 \\
\hline
\end{tabular}


MED_SHEAR) and low-shear (Fig. 4e and Table 1; referred to as LOW_SHEAR) runs, respectively. Additionally, two more simulations were performed using the CONTROL and LOW_SHEAR kinematic profiles where the Coriolis force was applied to the model perturbations (referred to as CONTROL_COR and LOW_SHEAR_COR, respectively) assuming an $f$-plane value of $8.882 \times 10^{-5} \mathrm{~s}^{-1}$, which corresponds to the latitude of Springfield, Missouri $\left(37.25^{\circ} \mathrm{N}\right)$.

Buoyant and dynamic components [i.e., all terms in Eq. (3)] of VPPGF were numerically solved for each run following the methods of Parker and Johnson (2004) and Coffer and Parker (2015) to investigate the wind shearinduced differences in the VPPGF. Briefly [see Eq. (3)], in this method, the buoyant pressure perturbation $p_{B}^{\prime}$, dynamic pressure perturbation $p_{D}^{\prime}$, and the dynamic linear pressure perturbation $p_{\mathrm{DL}}^{\prime}$ were numerically solved following the diagnostic equations presented in Wilhelmson and Ogura (1972) and Rotunno and Klemp (1982). Since the retrieval of the individual pressure perturbation terms required the inversion of a Laplacian (e.g., Rotunno and Klemp 1982, among others), the following boundary conditions were assumed (e.g., as in Coffer and Parker 2015): (i) the buoyant pressure perturbation $p_{B}^{\prime}$ satisfied the hydrostatic balance at the model boundaries; (ii) the dynamic pressure perturbation $p_{D}^{\prime}$ satisfied $p_{D}^{\prime}=p^{\prime}-p_{B}^{\prime}$ at the lateral boundaries, where $p^{\prime}$ is the pressure perturbation known from the model output; (iii) the dynamic linear pressure perturbation $p_{\mathrm{DL}}^{\prime}$ satisfied $p_{\mathrm{DL}}^{\prime}=0$ at the model boundaries; and (iv) the dynamic nonlinear portion of the perturbation pressure $p_{\mathrm{DNL}}^{\prime}$ was then treated as the residual of the dynamic pressure perturbation $p_{D}^{\prime}$ minus the dynamic linear pressure perturbation $p_{\mathrm{DL}}^{\prime}$ (i.e., $p_{\mathrm{DNL}}^{\prime}=p_{D}^{\prime}-p_{\mathrm{DL}}^{\prime}$ ). The resulting pressure perturbations were used to calculate the vertical accelerations associated with the various terms of the standard decomposition [e.g., those generalized in Eq. (4) and shown in Eq. (3)]. This analysis helps isolate the influence of the rotation on the overall strength of the updrafts and storm inflow through the calculation of the accelerations associated with VPPGF [i.e., terms in Eq. (3)], including those caused by $p_{\text {DNL }}^{\prime}$ [i.e., the spin term in Eq. (4)], the nonlinear dynamic vertical pressure perturbation force (NLD-VPPGF will refer to the accelerations induced by this term).

\section{Results: Non-Coriolis simulations}

The three simulations without Coriolis described above go on to produce convective systems that are similar in size, shape, and speed. Convection initiates from the forced convergence $2-3 \mathrm{~h}$ into the simulations, and all three simulations produce similar convective systems by $6 \mathrm{~h}$ into the simulations. Similar storms, from a simulated reflectivity point of view, develop in all three runs by time $t=9 \mathrm{~h}$ (Figs. 5a,c,e and simulation animations in the online supplemental material) and are maintained through the end of the simulations (see discussion below). All three runs produce the most intense convection in a fairly localized area on the southern and western flank of the storm, where low- to midlevel rotation is present in varying degrees of strength and maintenance depending on the specific simulation. A broad downshear stratiform region is present in all simulations, but slight variations in spatial extent are noticeable. The simulations produce similar radar depictions as the observed case described in section 2 (cf. Figs. 5a,c,e and 1a).

The convective systems produced in the simulations have spatial scales smaller than the $\mathrm{MCS}^{7}(\sim 80-100 \mathrm{~km}$ in spatial extent is seen for the CONTROL simulation) discussed in section 2 (cf. Figs. 1a,b and 5a,c,e). However, the simulations do contain embedded supercells, similar to the observed case (Figs. 1a,b). Because of the system longevity, nature of the initial forcing (i.e., elevated forced convergence), and the initially thermodynamically stable boundary layer, one could categorize the simulated systems as an MCS with embedded supercells.

By $t=11 \mathrm{~h}$ into each simulation, all three convective systems are continually back building (e.g., Schumacher and Johnson 2005) with the stratiform region still located downshear of the main convective region (Figs. 6a,c,e). Low- and midlevel rotation is present in all three cases but with varying strengths and longevity. Strong, sustained rotation and repeated mesocyclone development is seen in the CONTROL run (Figs. 6-8b), while comparatively weak, scattered, shallow, and short-lived rotations are seen in the LOW_SHEAR simulation (Figs. 6-8f). The MED_SHEAR run produces low-level rotation characteristics somewhere between CONTROL and LOW_SHEAR runs, in terms of strength and vortex longevity (Figs. 6-8d), which is not surprising given its $0-1-\mathrm{km}$ shear value lies between the other two runs. A small cold pool develops in each simulation after $t=9 \mathrm{~h}$ that is anchored to the region of most intense convection (Figs. 5b,d,f; and even shrinks by $t=11 \mathrm{~h}$ ). One hour later, a total of $t=13 \mathrm{~h}$ into the simulations, each simulation has a similar radar depiction (Figs. 7a,c,e); however, stronger, more persistent rotation is still maintained in the runs with higher

\footnotetext{
${ }^{7}$ The resulting convective systems simulated in the numerical runs do not all meet the strict length requirements often used for an MCS (e.g., $>35 \mathrm{dBZ}$ for $>100 \mathrm{~km}$; Parker and Johnson 2000).
} 

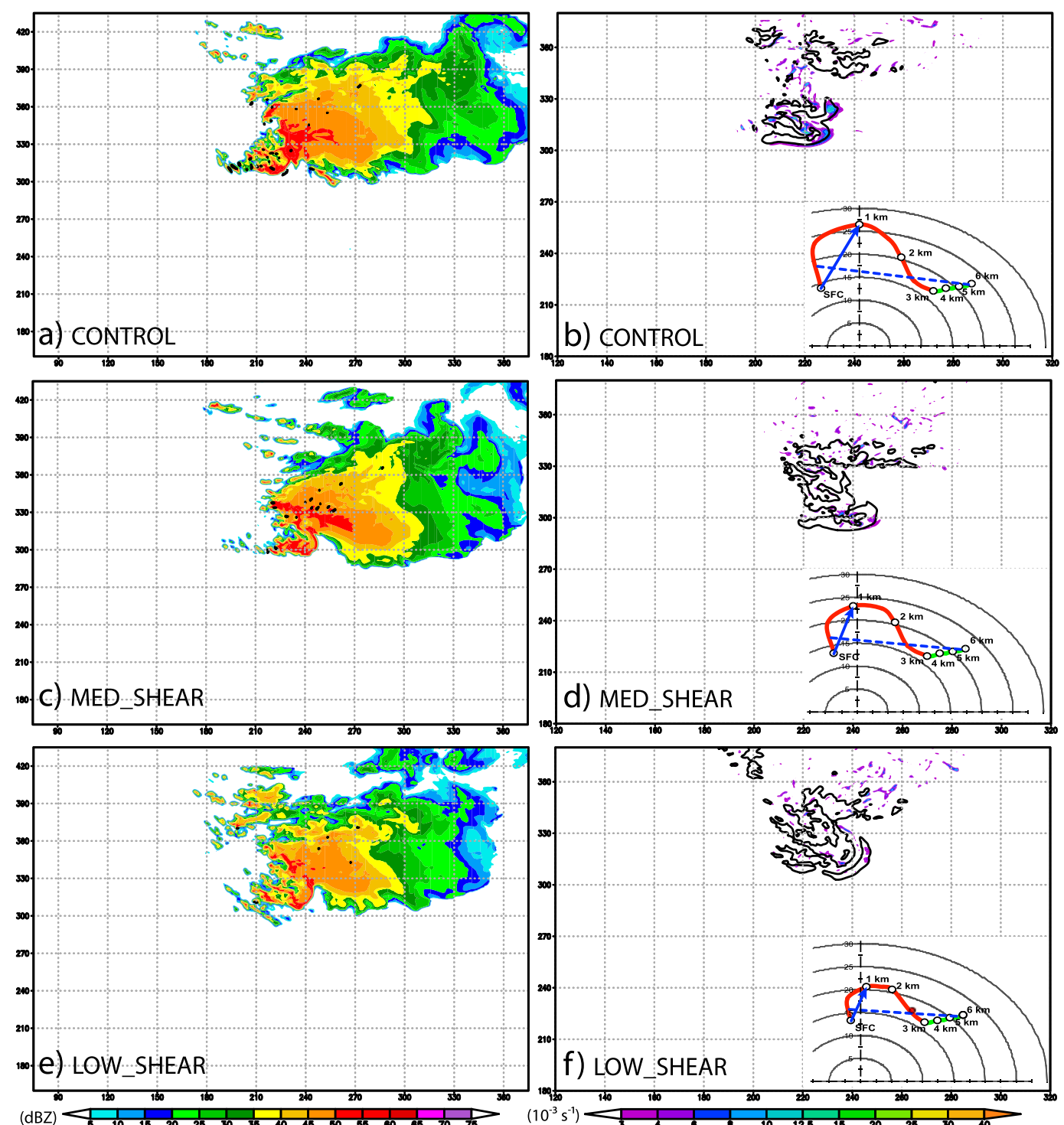

FIG. 5. (a),(c),(e) Simulated 1-km radar reflectivity (shaded; every $5 \mathrm{~dB} Z$ from 5 to $70 \mathrm{dBZ}$ ) and contoured 1-km vertical vorticity (black contours; starting at $10.0 \times 10^{-3} \mathrm{~s}^{-1}$ and plotted every $5.0 \times 10^{-3} \mathrm{~s}^{-1}$ ) valid $t=9 \mathrm{~h}$ into the simulations for the (a) CONTROL, (c) MED_SHEAR, and (e) LOW_SHEAR simulations. (b),(d), (f) Corresponding 500-m vertical vorticity $\left(\times 10^{-3} \mathrm{~s}^{-1}\right)$ and $500-\mathrm{m}$ perturbation potential temperature $\theta^{\prime}(-1.0 \mathrm{~K}$ contoured in black) for the (b) CONTROL, (d) MED_SHEAR, and (f) LOW_SHEAR simulations. Grid tick marks are in kilometers. Hodographs for each simulation are embedded in (b), (d), and (f), where the solid blue arrow represents $0-1-\mathrm{km}$ bulk wind difference, and dashed blue line represents boundary layer to $6-\mathrm{km}$ bulk wind difference.

0-1-km shear (Figs. 7b,d,f). A rather robust cold pool has developed in the MED_SHEAR run (Fig. 7d) and is beginning to develop in the LOW_SHEAR run (Fig. 7f) but not in the CONTROL simulation (Fig. 7b). At the end of the simulations (i.e., after $t=15 \mathrm{~h}$ of integration), the cold pools in the MED_SHEAR and LOW_SHEAR case have outrun the convection (Figs. 8d,f), which has resulted in limited low-level rotation (Figs. 8d,f) and weakened convection on the western flank of the storms.
The CONTROL run shows signs of continued, but slow, cold pool development and maintains low-level rotation throughout the simulations (Figs. 8a,b). The stratiform regions of the CONTROL and MED_SHEAR simulations are larger in spatial coverage and intensity compared to the LOW_SHEAR run through the last $2 \mathrm{~h}$ of both simulations (cf. Figs. 7a,c,e and 8a,c,e).

Substantially more precipitation occurred in terms of point maximum, areal mean, and areal coverage of large 

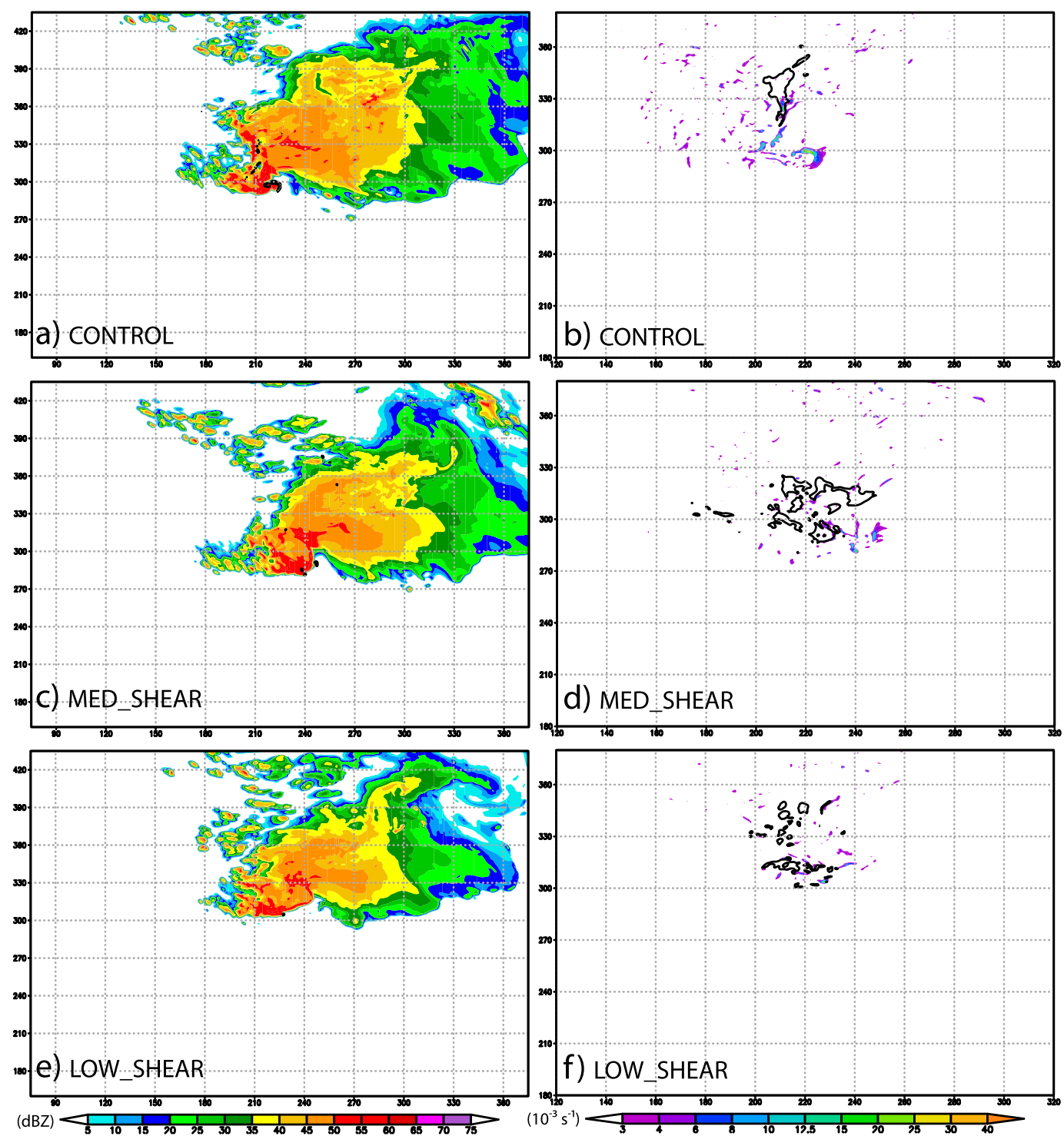

FIG. 6. As in Fig. 5, but valid $t=11 \mathrm{~h}$ into the simulations.

accumulations (e.g., 25, 50, $100 \mathrm{~mm}$; Table 2) for the CONTROL run compared to the MED_SHEAR and LOW_SHEAR simulations (Fig. 9, Table 2). The largest differences in accumulated precipitation between the runs appear in the areal coverage at the upper accumulation thresholds. The CONTROL run produces accumulation over $200 \mathrm{~mm}$ at multiple points, while the MED_SHEAR and LOW_SHEAR only have point maxima of 167 and $142 \mathrm{~mm}$, respectively (Fig. 9, Table 2). Furthermore, the amount of total accumulated precipitation and domain coverage at specific thresholds appears to increase with the magnitude of the low-level shear in the initial wind profile (Table 2). In other words, the accumulated precipitation for the three runs without Coriolis is larger in the simulations with more intense and prolonged low-level rotation, which is associated with the magnitude of the low-level shear. The increase in $0-1-\mathrm{km}$ shear between the simulations does lead to an increase in storm-relative inflow, since the approximate storm motion for each simulation is equal. Although this likely contributes to the increased precipitation, it does not explain the magnitude of the precipitation increases, likely because the stable boundary layer air would not reach its level of free convection by this process alone (see additional discussion on this matter in section 6).

Substantial differences in both mean (Figs. 10a-c) and maximum (Figs. 11a,c,e) vertical velocity were seen among the three simulations without Coriolis at the low levels. The average low-level vertical motion is larger and sustained for a longer period of time in the 

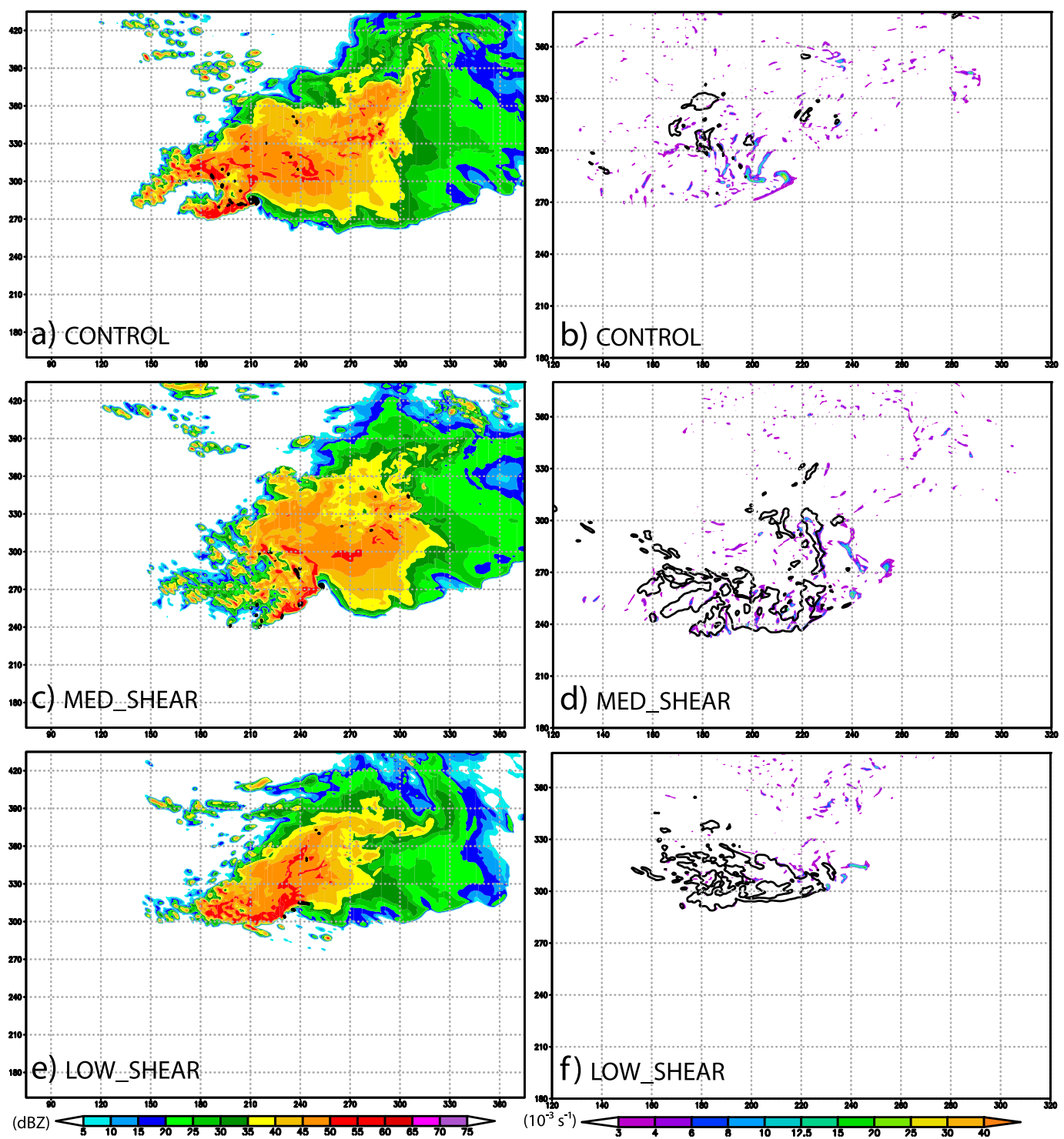

FIG. 7. As in Fig. 5, but valid $t=13 \mathrm{~h}$ into the simulations.

CONTROL simulation compared to the MED_SHEAR and LOW_SHEAR runs. The largest enhancements in the CONTROL run areal-mean vertical velocity over the other two simulations are seen at and above $1 \mathrm{~km}$ in height (cf. Figs. 10a-c). However, enhancements over the weaker-shear simulations are still seen in mean vertical velocity in the lowest levels (i.e., 300 and $500 \mathrm{~m}$ ) of the CONTROL run (cf. Figs. 10a-c). The CONTROL run continues to produce areal-mean positive low-level updrafts for $1 \mathrm{~h}$ over the LOW_SHEAR simulation and for $30 \mathrm{~min}$ over the MED_SHEAR run (cf. Figs. 10a-c). Furthermore, the CONTROL regularly produces stronger maximum updrafts (cf. Figs. 11a,c,e) than the other simulations with values approaching $20 \mathrm{~m} \mathrm{~s}^{-1}$ at $500 \mathrm{~m}$ and up to $40 \mathrm{~m} \mathrm{~s}^{-1}$ at $1.5 \mathrm{~km}$ (Fig. 11a).
Similarly, the CONTROL simulation produces higher maximum vertical vorticity values throughout the low levels both at individual times and in a mean maximum sense (i.e., the mean of the maximum values) compared to the MED_SHEAR and LOW_SHEAR runs (cf. Figs. 11b,d,f). The MED_SHEAR simulation, while showing relatively little difference in the areal-mean vertical velocity compared to LOW_SHEAR (cf. Figs. 11d,f), consistently produces higher maximum vertical motions throughout the low levels throughout the length of the simulation. All three runs show temporally sporadic but intense peaks in the maximum low-level vertical velocity (cf. Figs. 11a,c,e); however, the frequency and magnitude of these peaks are reduced from the CONTROL to the MED_SHEAR, and to the LOW_SHEAR runs (i.e., as 

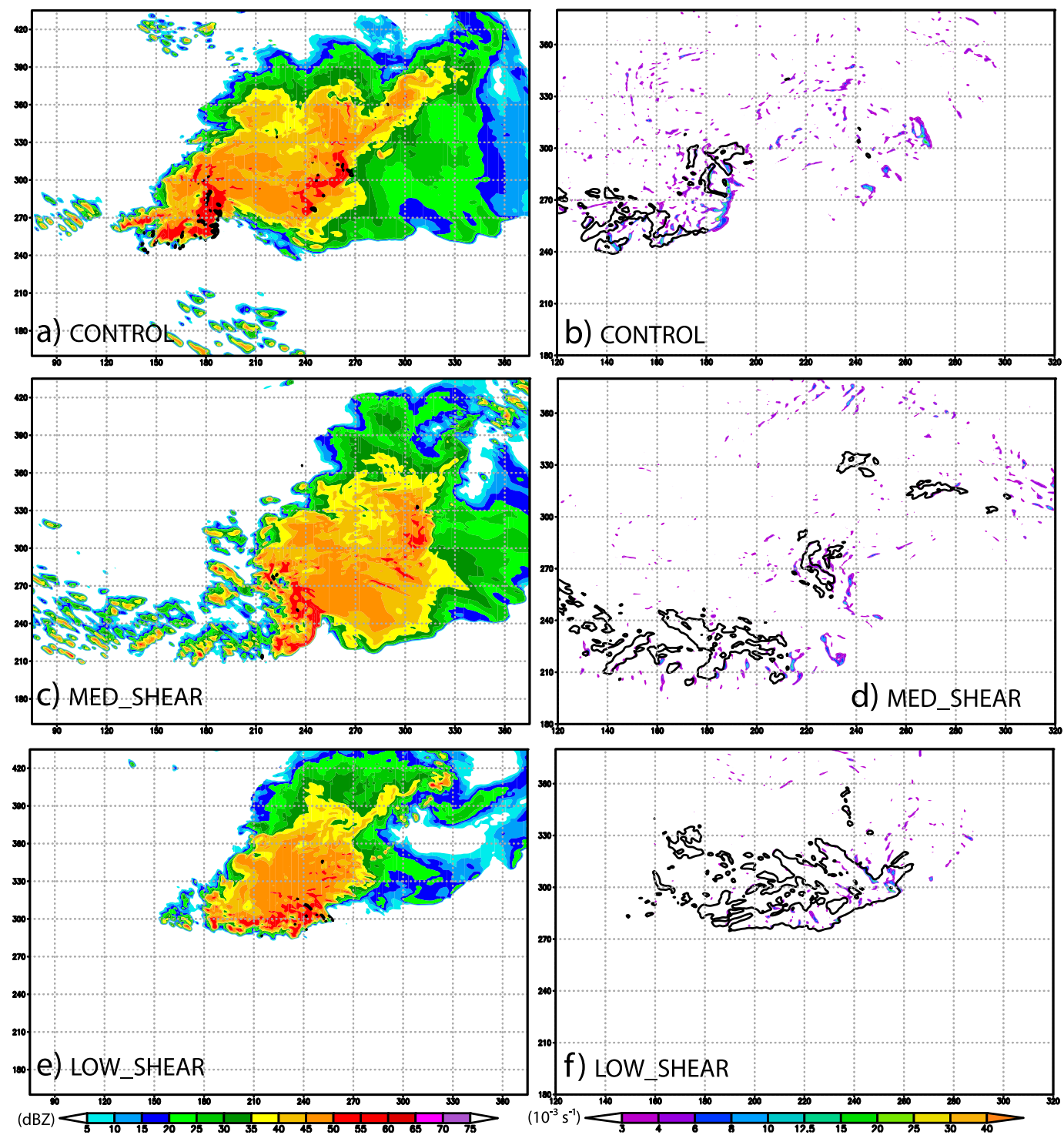

FIG. 8. As in Fig. 5, but valid $t=15 \mathrm{~h}$ into the simulations (i.e., the end).

you reduce the amount of $0-1-\mathrm{km}$ wind shear in the base-state profiles). The timing of the low-level maximum vertical velocities in all three runs is temporally correlated to periods of higher maximum vertical vorticity of similar duration (cf. Figs. 11a,c,e and 11b,d,f), where the more intense low-level vertical vorticity is associated with more intense vertical velocity. The temporal correlation and pulsing nature of the maximum low-level vertical velocity and vorticity is likely a manifestation of the pulsing nature of the low-level vortices that develop in each run, where the longevity and magnitude of the vortices are reduced with the amount of 0-1-km shear (cf. Figs. 6-8b,d,f; Figs. 11b,d, $\mathrm{f}$; and simulation animations in the online supplement).
The presence of such sustained and large vertical motions in the low levels of the simulation is worth noting, since there is substantial inhibition in the sounding for the surface-based parcel (i.e., Fig. 4a). However, all three simulated MCSs with embedded supercells are able to ingest parcels that originate below $750 \mathrm{~m}$ (low-level tracers), which contain moisture and CAPE, into the various updrafts (not shown). The concentrations of low-level tracers that reach $8 \mathrm{~km}$ do not differ substantially between the three runs, but the mean concentration is ordered to the amount of lowlevel shear in each simulation (not shown).

Large differences are seen in the low-level total positive vertical mass flux from updrafts (Fig. 12). A substantial 
TABLE 2. Modeled rainfall accumulation statistics for simulations performed in this study.

\begin{tabular}{|c|c|c|c|c|c|}
\hline Statistic & CONTROL & MED_SHEAR & LOW_SHEAR & CONTROL_COR & LOW_SHEAR_COR \\
\hline Mean areal accumulation $\left(\mathrm{mm} \mathrm{km}^{-2}\right)$ & 3.10 & 2.43 & 1.95 & 2.03 & 1.75 \\
\hline Max accumulation $(\mathrm{mm})$ & 220 & 167 & 142 & 222 & 132 \\
\hline $\begin{array}{l}\text { Coverage of at least } 25-\mathrm{mm} \\
\text { accumulation }(\%)\end{array}$ & 5.66 & 5.05 & 3.88 & 4.17 & 3.77 \\
\hline $\begin{array}{l}\text { Coverage of at least } 50-\mathrm{mm} \\
\text { accumulation }(\%)\end{array}$ & 3.75 & 2.90 & 2.36 & 1.83 & 2.30 \\
\hline $\begin{array}{l}\text { Coverage of at least } 100-\mathrm{mm} \\
\text { accumulation }(\%)\end{array}$ & 1.19 & 0.42 & 0.36 & 0.60 & 0.33 \\
\hline $\begin{array}{l}\text { Coverage of at least } 150-\mathrm{mm} \\
\text { accumulation }(\%)\end{array}$ & 0.27 & 0.02 & - & 0.20 & - \\
\hline $\begin{array}{l}\text { Coverage of at least } 200-\mathrm{mm} \\
\text { accumulation }(\%)\end{array}$ & 0.009 & - & - & 0.02 & - \\
\hline
\end{tabular}

and noteworthy increase in low-level positive vertical mass flux is seen for the CONTROL simulation versus the MED_SHEAR and LOW_SHEAR runs (Fig. 12) by the end of the simulations. These differences, especially between the CONTROL and LOW_SHEAR runs, are maintained through all levels (Figs. 12b-d) with the vertical mass flux at each vertical level ordered to the amount of low-level shear (and the amount of low-level vertical rotation; Fig. 10) in each simulation (i.e., higher $0-1-\mathrm{km}$ shear has higher vertical mass flux; Fig. 12). These vertical mass flux differences illustrate the net effect of the large low-level vertical velocity differences seen between each simulation (cf. Figs. 10 and 12). The differences in vertical mass flux are greatest between the CONTROL and LOW_SHEAR simulations. The MED_SHEAR run maintains vertical mass flux values that are much closer in magnitude to the CONTROL simulation above the lowest levels (Fig. 12), but both simulations maintain substantially larger vertical mass flux values when compared to the LOW_SHEAR run. The large reduction in vertical mass flux near $t=13 \mathrm{~h}$ at low levels in the MED_SHEAR (Figs. 12a,b) run is likely due to the cold pool undercutting the most intense, rotation-containing convection on the southern flank of the storm (Fig. 7c). The MED_SHEAR and LOW_SHEAR runs develop cold pools with horizontal scales matching that of the storm itself and maximum $\theta^{\prime}$ depressions at $500 \mathrm{~m}$ of $\theta^{\prime} \sim-2.5 \mathrm{~K}$, while the cold pool in the CONTROL simulation is weaker, maximum $\theta^{\prime}$ depressions at $500 \mathrm{~m}$ from $\theta^{\prime} \sim-1.5$ to $-2.0 \mathrm{~K}$, and has less spatial extent (see Figs. 6-8). Considering the greater rainfall in CONTROL (and thus larger quantity of hydrometeors), it is unclear why the cold pool remains weak compared to the lower-shear runs.

Given that intense updrafts are present at and below $500 \mathrm{~m}$ in the CONTROL, MED_SHEAR, and LOW_SHEAR. runs and that these updrafts are ingesting high concentrations of passive tracers from within an inhibited boundary layer, the horizontal characteristics of the VPPGF at or near $500 \mathrm{~m}$ were a focus of the pressure retrieval diagnostics. While VPPGF accelerations are present at other vertical levels in the simulations, the nature of the rotation (i.e., largest in lower levels) leads to the largest VPPGF being found in low levels. The analysis was mainly accomplished by creating translated swaths (i.e., map views from $t=9$ to $15 \mathrm{~h}$ where the plotting accounts for the numerical model domain translation speed) of maximum dynamic forcing terms at $500 \mathrm{~m}$ at any grid point, which can be interpreted similarly to maximum updraft helicity swaths (e.g., Clark et al. 2013) used in severe storm forecasting (e.g., Figs. 13a,c,e). The resulting analysis shows that intense, persistent low-level acceleration associated with the NLD-VPPGF is present in the CONTROL run but is continually less persistent and intense in the MED_ SHEAR and LOW_SHEAR simulations (cf. Figs. 13a,c,e). The signature of individual rotating updrafts and cyclic mesocyclogenesis (e.g., Adlerman et al. 1999) can be seen in all simulations but is especially noticeable for a couple instances in the LOW_SHEAR run (Fig. 13e). This reinforces the notion that the CONTROL run (and to some extent the MED_SHEAR simulation) maintains persistent rotation and the associated low-level acceleration, which is comparatively intense (cf. Figs. 13a,c,e), over a large portion of the domain, as opposed to the few brief isolated spinups seen in the LOW_SHEAR run. When both acceleration from the NLD-VPPGF and the linear dynamic VPPGF [see Eq. (3)] is taken into account to create the ACCD, a very similar low-level acceleration to that associated with the NLD-VPPGF is seen (cf. fill contours in Figs. 13a,c,e and contours in Figs. 13b,d,f). This implies that the NLD-VPPGF dominates the total low-level dynamic acceleration in these simulations. 

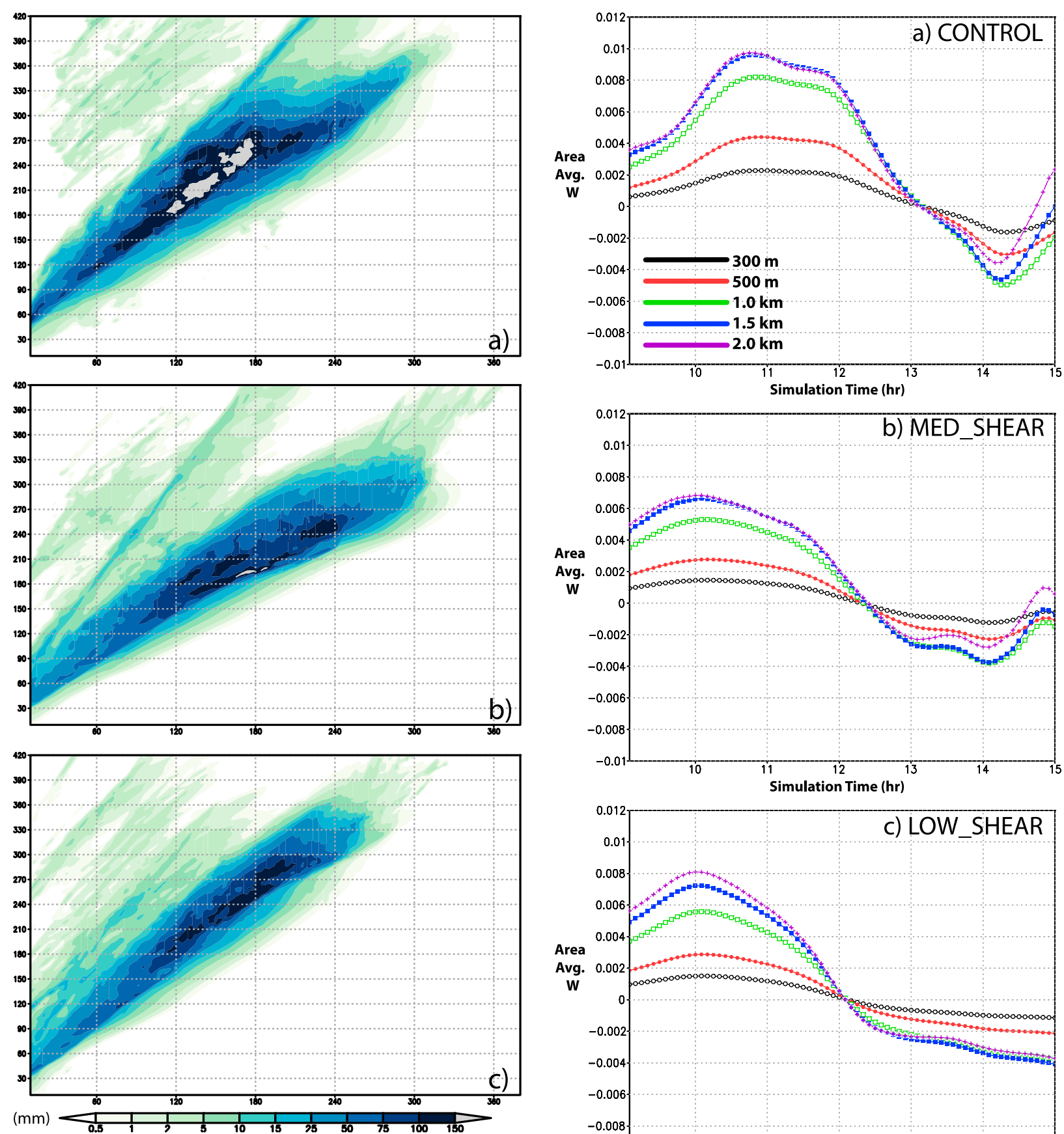

FIG. 9. Total accumulated precipitation ( $\mathrm{mm}$ ), accounting for the translation of the numerical model domain, in (a) CONTROL, (b) MED_SHEAR, and (c) LOW_SHEAR simulations. Runspecific statistics are presented in Table 2. Axis tick marks are in kilometers.

The influence of the low-level ACCD on the low-level vertical velocity field is quite apparent in the nonCoriolis simulations (Figs. 13b,d,f). A clear correlation, especially in the CONTROL run, exists between the most intense low-level updrafts and the location of

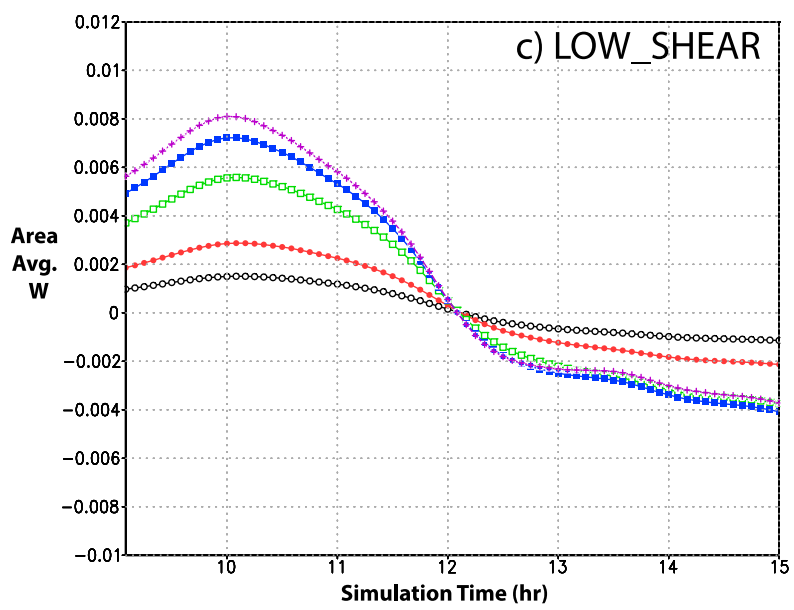

FIG. 10. Time series of area-averaged vertical motion $\left(\mathrm{m} \mathrm{s}^{-1} \mathrm{~m}^{-2}\right)$ for the (a) CONTROL, (b) MED_SHEAR, and (c) LOW_SHEAR simulations at model height levels of $300 \mathrm{~m}$ (black), $500 \mathrm{~m}$ (red), $1.0 \mathrm{~km}$ (green), $1.5 \mathrm{~km}$ (blue), and $2.0 \mathrm{~km}$ (purple). The areal averaging was performed over the spatial extent of the model domain depicted in Figs. 5-8.

the greatest ACCD. This shows the importance of the ACCD in getting intense, in some cases up to $20 \mathrm{~m} \mathrm{~s}^{-1}$, updrafts near the surface (i.e., $500 \mathrm{~m}$ ) when the parcels 

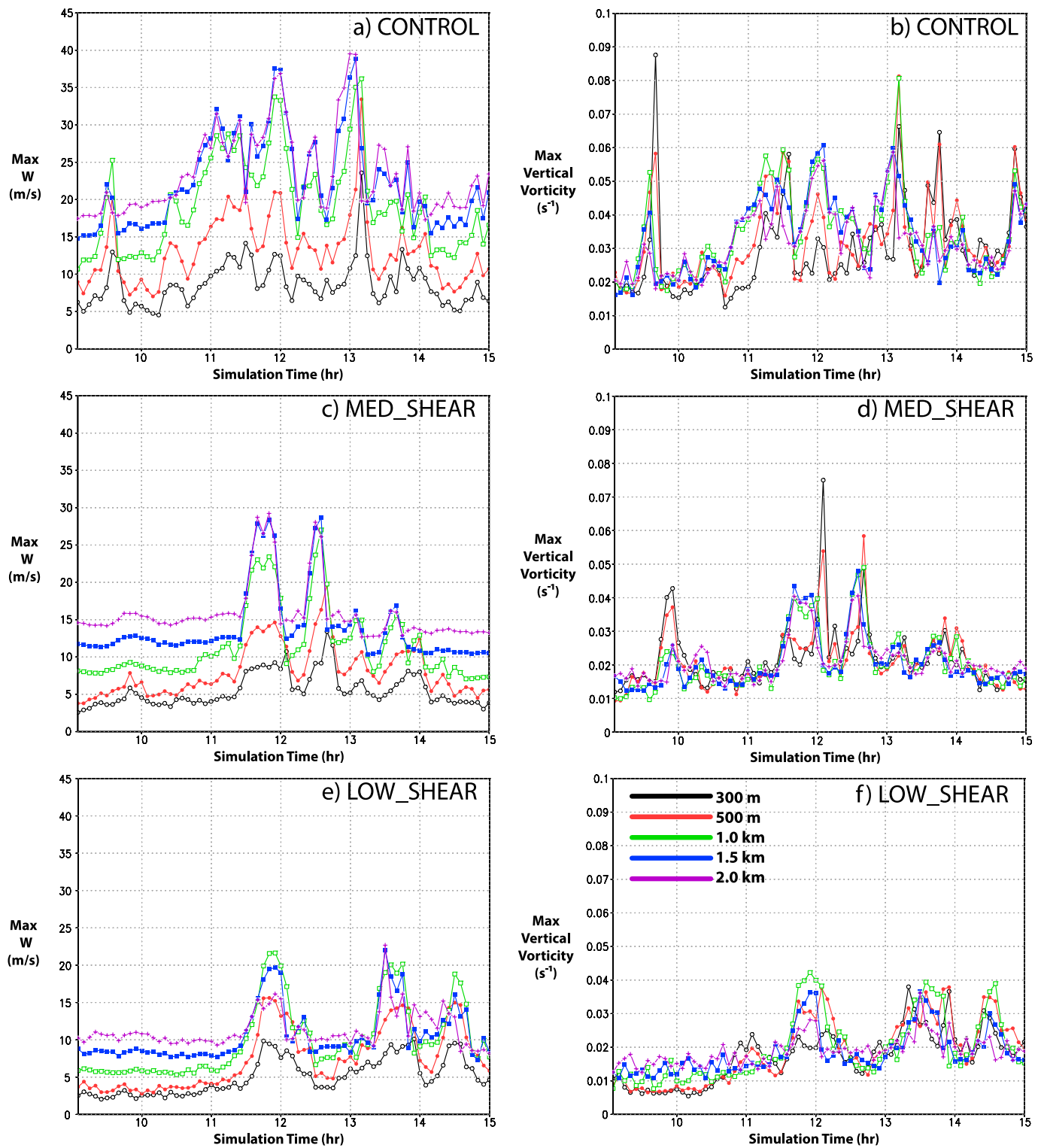

FIG. 11. Time series of maximum updraft velocity $w\left(\mathrm{~m} \mathrm{~s}^{-1}\right)$ for the (a) CONTROL, (c) MED_SHEAR, and (e) LOW_SHEAR runs at model height levels of $300 \mathrm{~m}$ (black), $500 \mathrm{~m}$ (red), $1.0 \mathrm{~km}$ (green), $1.5 \mathrm{~km}$ (blue), and $2.0 \mathrm{~km}$ (purple). Time series of maximum vertical vorticity $\left(\mathrm{s}^{-1}\right)$ for the (b) CONTROL, (d) MED_SHEAR, and (f) LOW_SHEAR runs at the same model height as (a), (c), and (e).

themselves are conditionally stable. This is further reinforced by the relative lack of ACCB [see Eq. (3)] of a similar magnitude in any of the simulations near the surface (e.g., lack of color contours in Figs. 14b,d,f). The extent and magnitude of the 500-m updrafts between the CONTROL, MED_SHEAR, and LOW_SHEAR runs increases with the extent and magnitude of the NLD-VPPGF at that same level, which also intensifies with the amount of $0-1-\mathrm{km}$ shear in the base-state kinematic profile of each simulation. The highest low-level total condensate mixing ratios (QTOTs; e.g.,
Figs. 14a,c,e) are offset from the region of largest ACCD (and corresponding updrafts) with the breadth and maximum of the condensate values increasing with $0-1-\mathrm{km}$ shear values.

The vertical extent and magnitude of the NLD-VPPGF is maximized in the CONTROL simulation (cf. Figs. 15a,c,e) with diminishing values in both depth and intensity when moving sequentially to the lower-shear runs (i.e., to MED_SHEAR and LOW_SHEAR, respectively). This in turn leads to more intense updrafts closer to ground level as the low-level shear increases (and, 
300-m Vertical Mass Flux

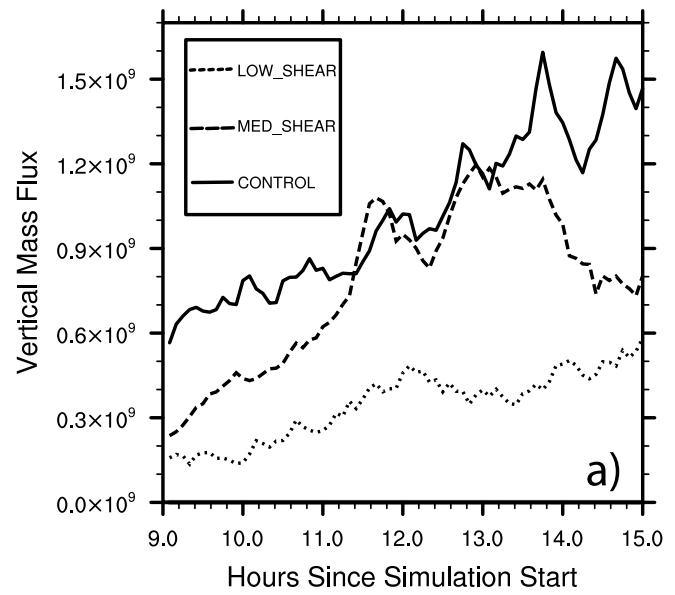

1.0-km Vertical Mass Flux

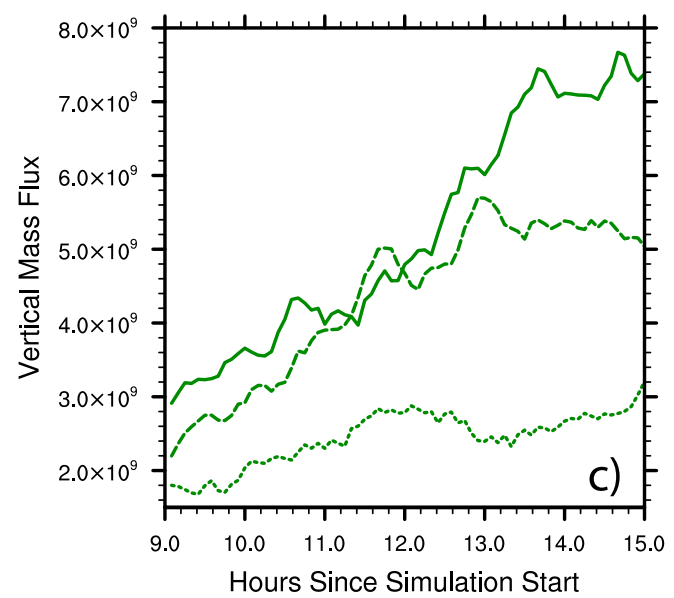

2.0-km Vertical Mass Flux

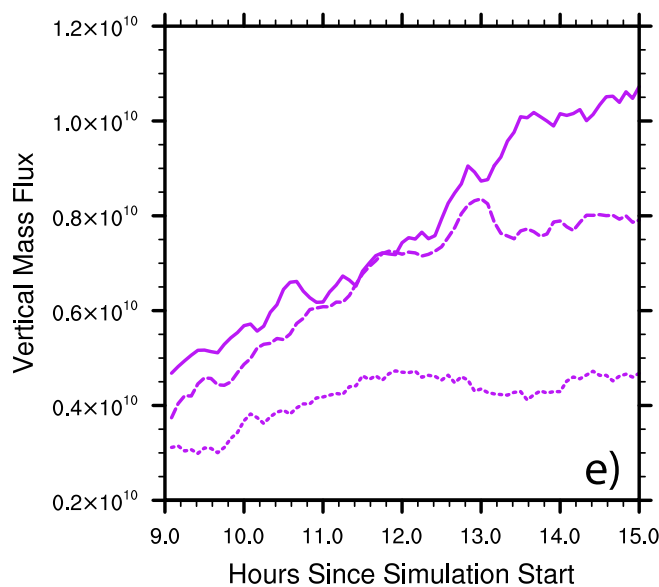

500-m Vertical Mass Flux

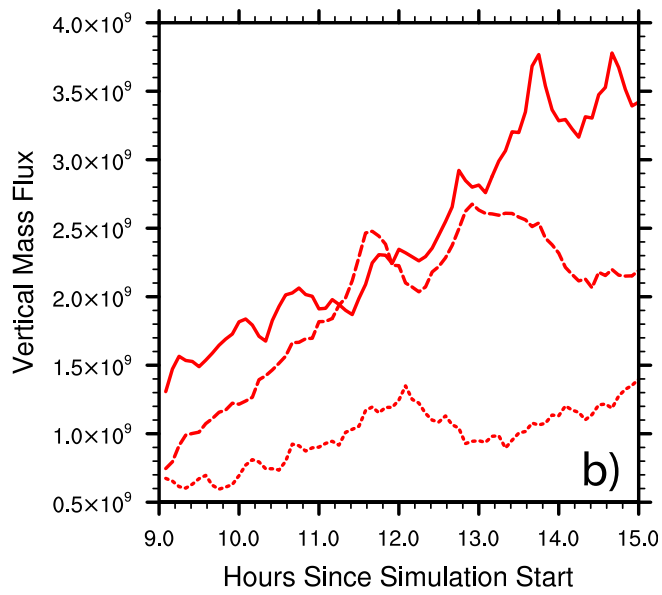

.5-km Vertical Mass Flux

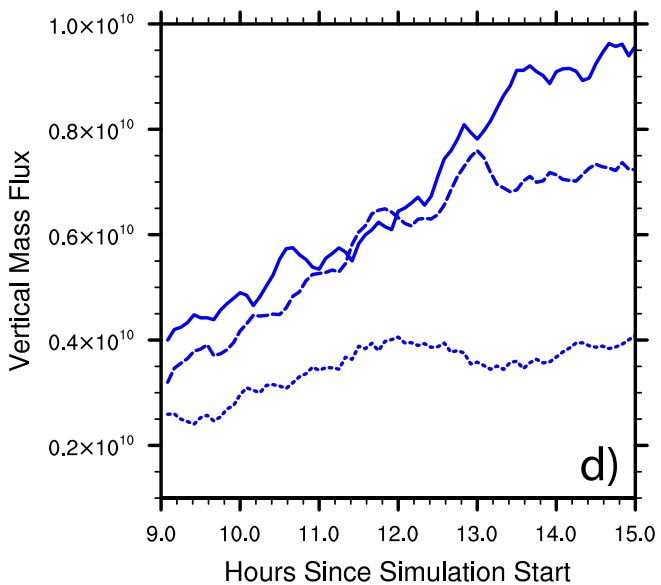

8.0-km Vertical Mass Flux

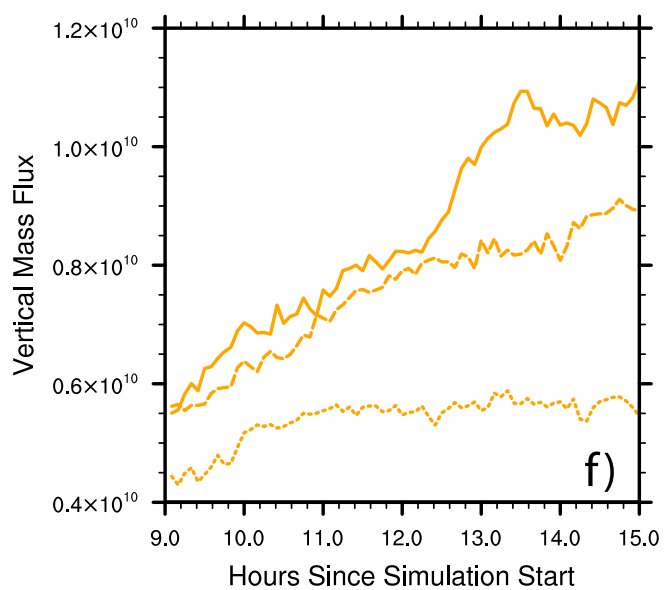

FIG. 12. Total vertical mass flux ( $\mathrm{g} \mathrm{s}^{-1}$ ) of updrafts with magnitude over $1 \mathrm{~m} \mathrm{~s}^{-1}$ over the portion of the domain containing the modeled MCS at $z=$ (a) $300 \mathrm{~m}$, (b) $500 \mathrm{~m}$, (c) $1.0 \mathrm{~km}$, (d) $1.5 \mathrm{~km}$, (e) $2.0 \mathrm{~km}$, and (e) $8.0 \mathrm{~km}$ for the CONTROL (solid line), MED_SHEAR (long dashed line), and LOW_SHEAR (short dashed line) runs. The scale of the ordinate is different in each panel. The areal averaging was performed over the spatial extent of the model domain depicted in Figs. 5-8. 

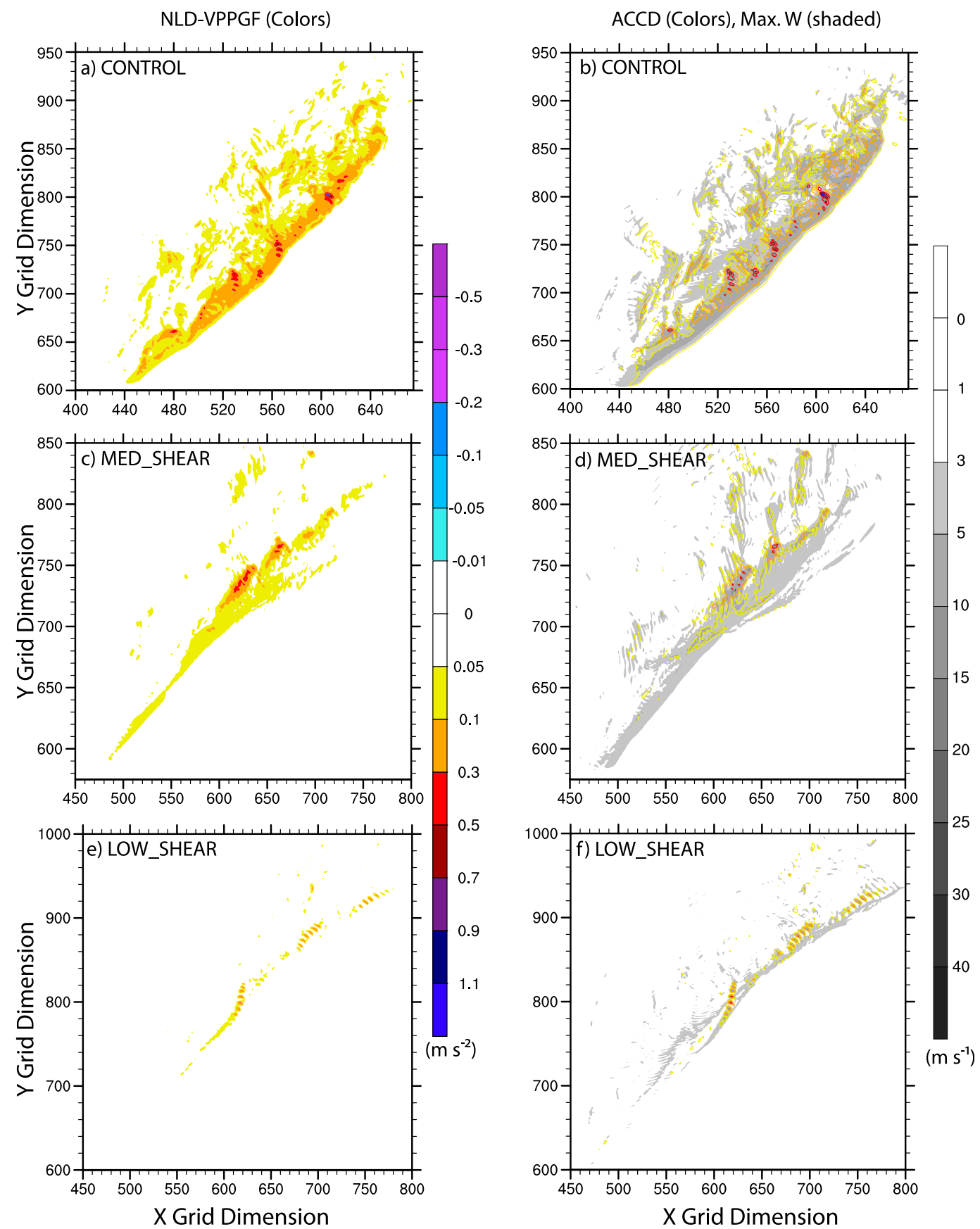

FIG. 13. (a),(c),(e) Translated swaths of maximum 500-m dynamic nonlinear vertical perturbation pressure gradient acceleration (NLD-VPPGF; shaded; $\mathrm{m} \mathrm{s}^{-2}$ ) valid from $t=9$ to $15 \mathrm{~h}$ into the (a) CONTROL, (c) MED_SHEAR, and (e) LOW_SHEAR simulations. (b),(d),(f) Translated swaths of maximum 500-m vertical velocity (shaded; $\mathrm{m} \mathrm{s}^{-1}$ ), with maximum 500-m ACCD [contoured; colors match fill contour values in (a), (c), and (e)] overlaid for the (b) CONTROL, (d) MED_SHEAR, and (f) LOW_SHEAR runs valid from $t=9$ to $15 \mathrm{~h}$ into the simulations. Axes depict model grid points where grid spacing between points is $500 \mathrm{~m}$.

with that, the low-level rotation) with time-mean 10 , 5 , and $3 \mathrm{~ms}^{-1}$ updrafts sustained below $1 \mathrm{~km}$ for the CONTROL, MED_SHEAR, and LOW_SHEAR runs, respectively (Figs. 15a,c,e). The cross sections show that ACCB is present in the low levels of each simulation, but the largest values are maximized above $2 \mathrm{~km}$ in height (Figs. 16a,c,e) with little to no positive (in most cases negative) ACCB present in the lowest 

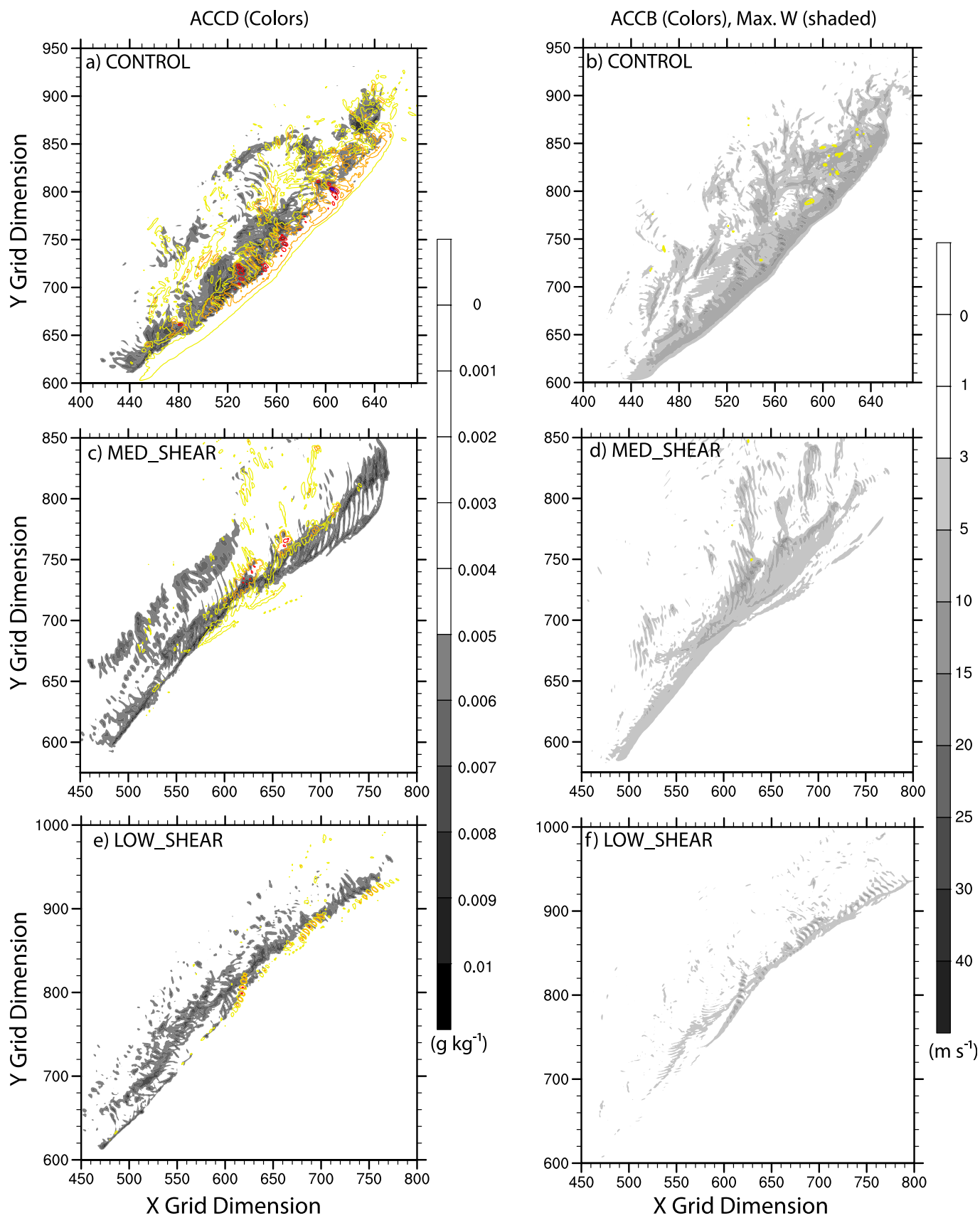

FIG. 14. (a),(c),(e) Translated swaths of maximum 500-m maximum total condensate mixing ratio (shaded; $\mathrm{g} \mathrm{kg}^{-1}$ ), with maximum 500-m ACCD (contoured; colors match fill contour values in Figs. 13a,c,e) overlaid for the (a) CONTROL, (c) MED_SHEAR, and (e) LOW_SHEAR runs valid from $t=9$ to $15 \mathrm{~h}$ into the simulations. (b),(d),(f) Translated swaths of maximum 500-m vertical velocity $w$ (shaded; $\mathrm{m} \mathrm{s}^{-1}$ ), with maximum 500-m total buoyant acceleration (contoured; colors match fill contour values in Figs. 13a,c,e) overlaid for the (b) CONTROL, (d) MED_SHEAR, and (f) LOW_SHEAR runs valid from $t=9$ to $15 \mathrm{~h}$ into the simulations. Axes depict model grid points where grid spacing between points is $500 \mathrm{~m}$.

levels. Additionally, the ACCB that is present is an order of magnitude less than the acceleration associated with the NLD-VPPGF, which is not necessarily surprising given the nature of the forcing and the initial thermodynamic profile. Mean low-level total condensate mixing ratios also increase with increasing mean low-level updraft strength (Figs. 16b,d,f), which intensifies with the amount of low-level acceleration form 
Vertical Velocity
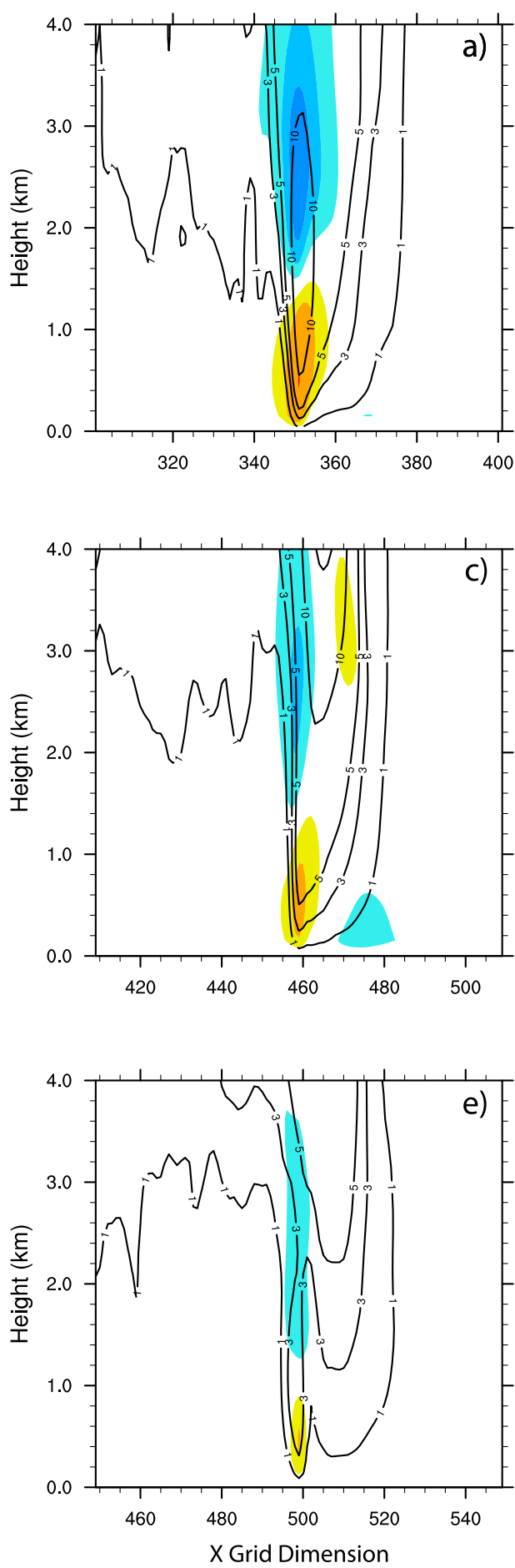

Vertical Vorticity

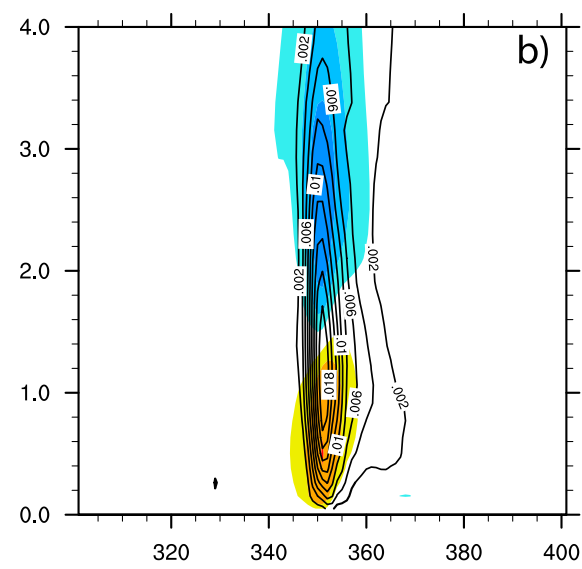

O)
응

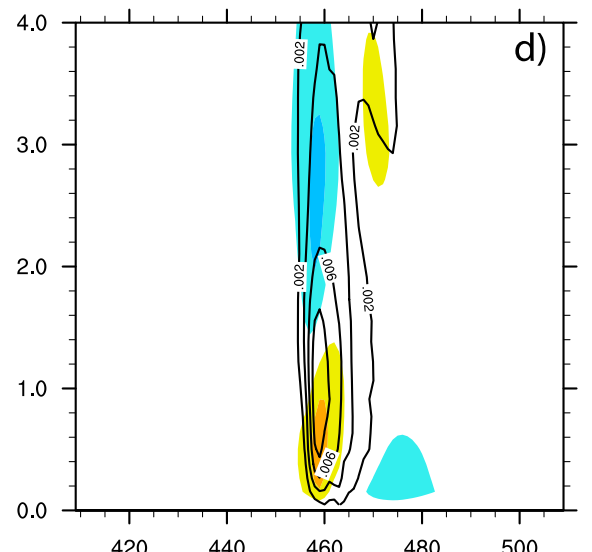

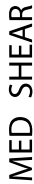

$\left(\mathrm{m} \mathrm{s}^{-2}\right)$
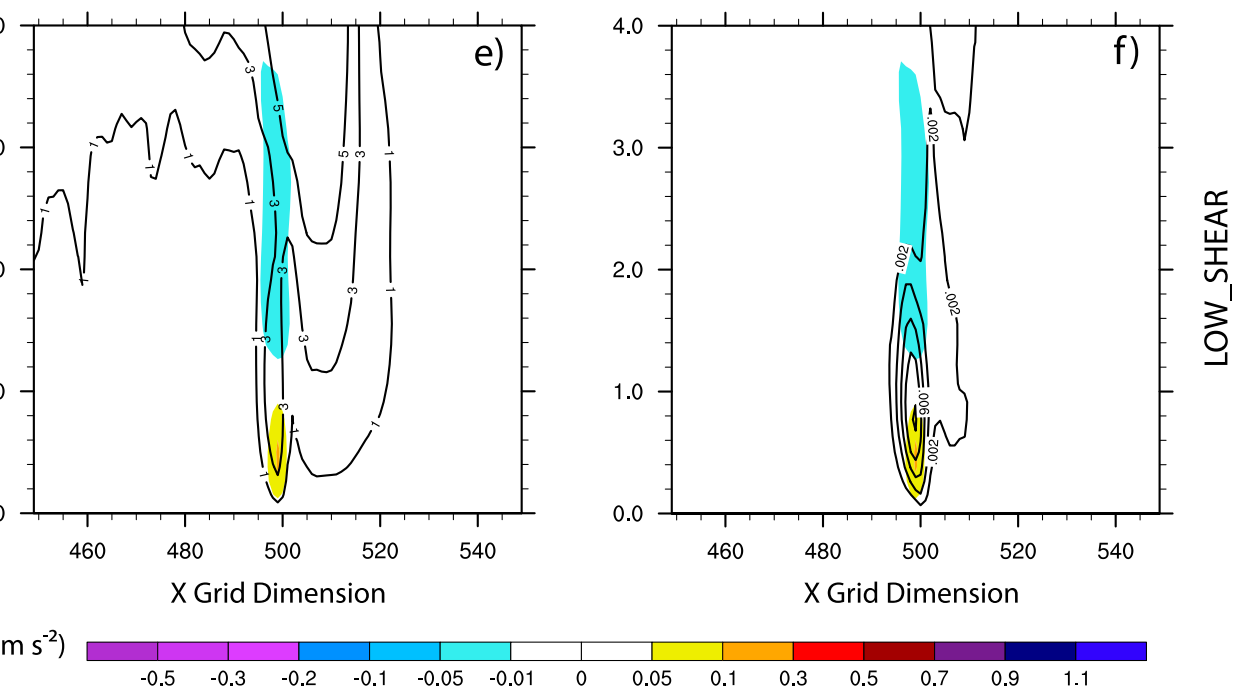

FIG. 15. Time-mean east-west cross sections through the point of maximum 500-m NLD-VPPGF acceleration (fill contour; $\mathrm{m} \mathrm{s}^{-2}$ ) overlaid with the corresponding mean (a),(c),(e) vertical velocity (contoured at 1 and $3 \mathrm{~m} \mathrm{~s}^{-1}$, then every $5 \mathrm{~m} \mathrm{~s}^{-1}$ above $5 \mathrm{~m} \mathrm{~s}^{-1}$ ) and (b),(d),(f) vertical vorticity (contoured every $5 \times 10^{-3} \mathrm{~s}^{-1}$ above $5 \times 10^{-3} \mathrm{~s}^{-1}$ ) for the (a),(b) CONTROL, (c),(d) MED_SHEAR, and (e),(f) LOW_SHEAR simulations. The $x$ axis depicts model grid points where grid spacing between points is $500 \mathrm{~m}$. 
ACCB
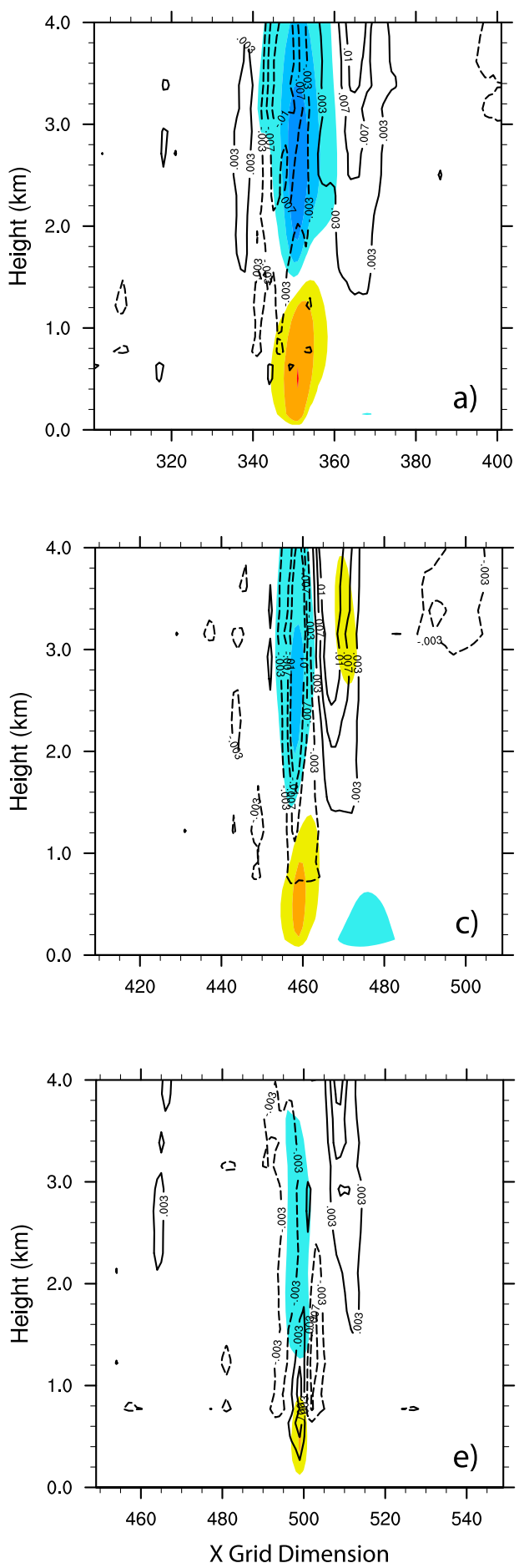

QTOT
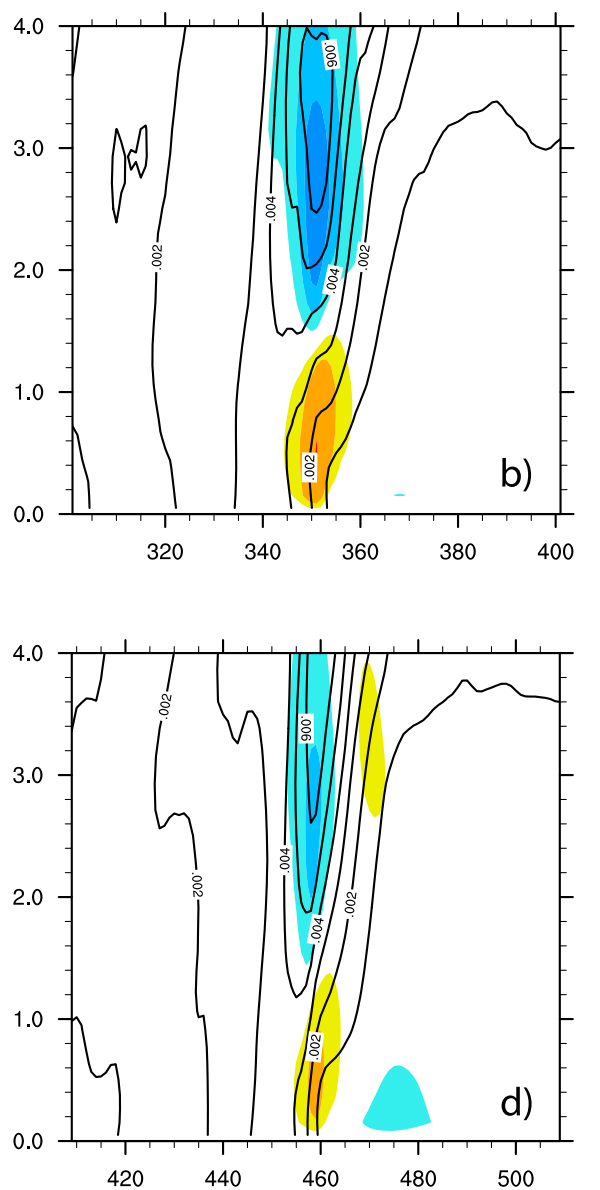

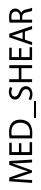

$\left(\mathrm{m} \mathrm{s}^{-2}\right)$

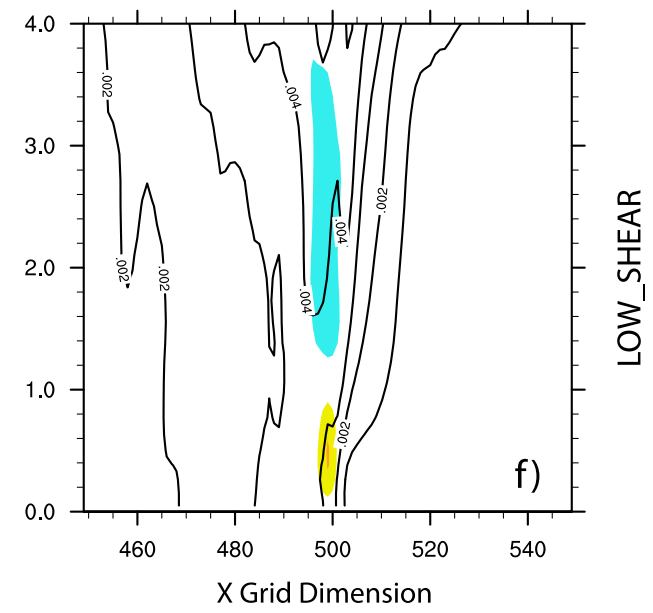

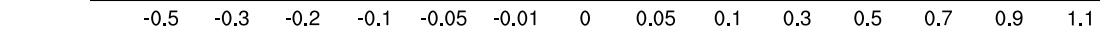

FIG. 16. Time-mean east-west cross sections through the point of maximum 500-m NLD-VPPGF acceleration (fill contour; $\mathrm{m} \mathrm{s}^{-2}$ ) overlaid with the corresponding (a),(c),(e) ACCB [solid contours (dashed) positive (negative) values at $\pm 0.003, \pm 0.007$, and $\pm 0.01 \mathrm{~m} \mathrm{~s}^{-2}$ ] and (b), (d), (e) QTOT (contoured every $0.01 \mathrm{~g} \mathrm{~kg}^{-1}$ )for the (a),(b) CONTROL, (c),(d) MED_SHEAR, and (e),(f) LOW_SHEAR simulations. The $x$ axis depicts model grid points where grid spacing between points is $500 \mathrm{~m}$. 
the NLD-VPPGF and $0-1-\mathrm{km}$ vertical wind shear. The more intense lower updrafts lead to increased volume of higher mean total condensate values (i.e., the breadth of higher-magnitude mean total condensate contours is larger at a given level) lower in the atmospheric column in the higher-shear runs (Figs. 16b,d,f). This seems to show the enhancement of precipitation formation processes by the NLD-VPPGF forced lowlevel updrafts in the runs with higher low-level vertical wind shear.

While the pressure decomposition undertaken here does not explicitly separate the NLD-VPPGF term into the accelerations associated with spin and those associated with deformation [i.e., the splat term in Eq. (4)], the terms do produce oppositely signed pressure perturbations. The vertical low-level accelerations presented in this manuscript are largely associated with negative nonlinear dynamic pressure perturbations (not shown), which implies that the spin term is dominating over the deformation term in these dynamically forced updrafts. Persistent low- to midlevel vertical rotation is present at the location of maximum NLD-VPPGF acceleration in all three simulations (Figs. 15b,d,f). The vertical depth and mean magnitude of the vertical rotation increases with the increase in low-level shear through the simulations (cf. Figs. 15b,d,f). Correspondingly, the magnitude and depth of the positive acceleration associated with the NLD-VPPGF increases with increasing vertical vorticity (fill colors in Figs. 15b,d,f). These spatial relationships hold in the mean sense (i.e., Figs. 15b,d,f) but also are seen in the regular temporal and spatial collocation of vertical vorticity and NLD-VPPGF associated acceleration at individual times throughout all three of the simulations (see supplemental material for animations).

The continued collocation of the NLD-VPPGF acceleration and vertical vorticity, in the bulk sense (i.e., described by the mean cross sections), support the idea that the spin portion of the NLD-VPPGF is playing the primary role in enhancing the low-level acceleration and, thus, updrafts. Specifically examining the CONTROL simulation during the mature phase of the storm (i.e., Fig. 17 at $t=11 \mathrm{~h} 55 \mathrm{~min}$ ), the main stormscale region of surface-based vertical motion is collocated with the region of significant vertical vorticity and NLD-VPPGF acceleration (Figs. 17a,c), where, as the pressure perturbation theory suggests, the NLD-VPPGF accelerations are maximized below the levels of maximum rotation. In this region where the embedded supercells are present, $w$ exceeds $15 \mathrm{~m} \mathrm{~s}^{-1}$ within the originally stable boundary layer, just above the regions of most intense NLD-VPPGF accelerations, which is collocated with regions of intense rotation around the vertical axis (Figs. 17a,c). The mid- to upper-level updrafts are also maximized above the low-level regions of NLD-VPPGF associated acceleration (Fig. 17c). Lifting along the cold pool at this time in the CONTROL run is much shallower and weaker (Figs. 17a,b). While very weak acceleration, compared to the regions where rotation is present, associated with the NLD-VPPGF is seen, the resulting combination of this lift and traditional cold pool lifting results in a shallow updraft that does not extend through the midlevels (Fig. 17b). While this lifting along the cold pool edge is more persistent in other simulations (not shown), it is regularly weaker and more elevated than that associated with rotating updrafts, because of the enhancement of the NLD-VPPGF. Additionally, the theta perturbations (Figs. 17b,c) appear to be elevated off the surface, centered largely near $1 \mathrm{~km}$, which is likely a results of the stability in the low levels of the initial thermodynamic profile. This hints that gravity wave processes might be acting along with the cold pool to lift parcels along the flanks of the system (as in Schumacher 2009).

\section{Results: Coriolis simulations}

When the Coriolis force is taken into account for kinematic profiles with the highest (i.e., CONTROL_COR) and lowest (i.e., LOW_SHEAR_COR) 0-1-km shear, very little change is seen in the MCS's evolution compared to the runs without the inclusion of the Coriolis force. The CONTROL_COR and LOW_SHEAR_COR both produce a back-building-type MCS (Figs. 18a,b) similar in both spatial appearance and the low-level vortex characteristics to the non-Coriolis simulations (cf. Figs. 18c,d and Fig. 7). However, the runs that include Coriolis produce less mean areal precipitation but similar maximum values when compared to the runs without Coriolis (Table 2). This seems to be most likely associated with the CONTROL_COR and LOW_SHEAR_COR runs producing smaller convective systems (cf. Figs. 18a, b and Figs. 7a,e). The differing system sizes can likely be exampled by the Coriolis simulations having a finite Rossby radius, compared to an infinite Rossby radius in the simulations without Coriolis.

Stronger, more sustained rotation is seen in the CONTROL_COR run compared to the LOW_SHEAR_ COR simulation (brief snapshot presented in Figs. 18c,d). Similar to the CONTROL and LOW_SHEAR runs, the CONTROL_COR and LOW_SHEAR_COR simulations produce a very weak but quite extensive cold pool, respectively, by $t=13 \mathrm{~h}$ into the model integration (Figs. 18a,b). Further, the CONTROL_COR run produces more run accumulated total precipitation, areal average 

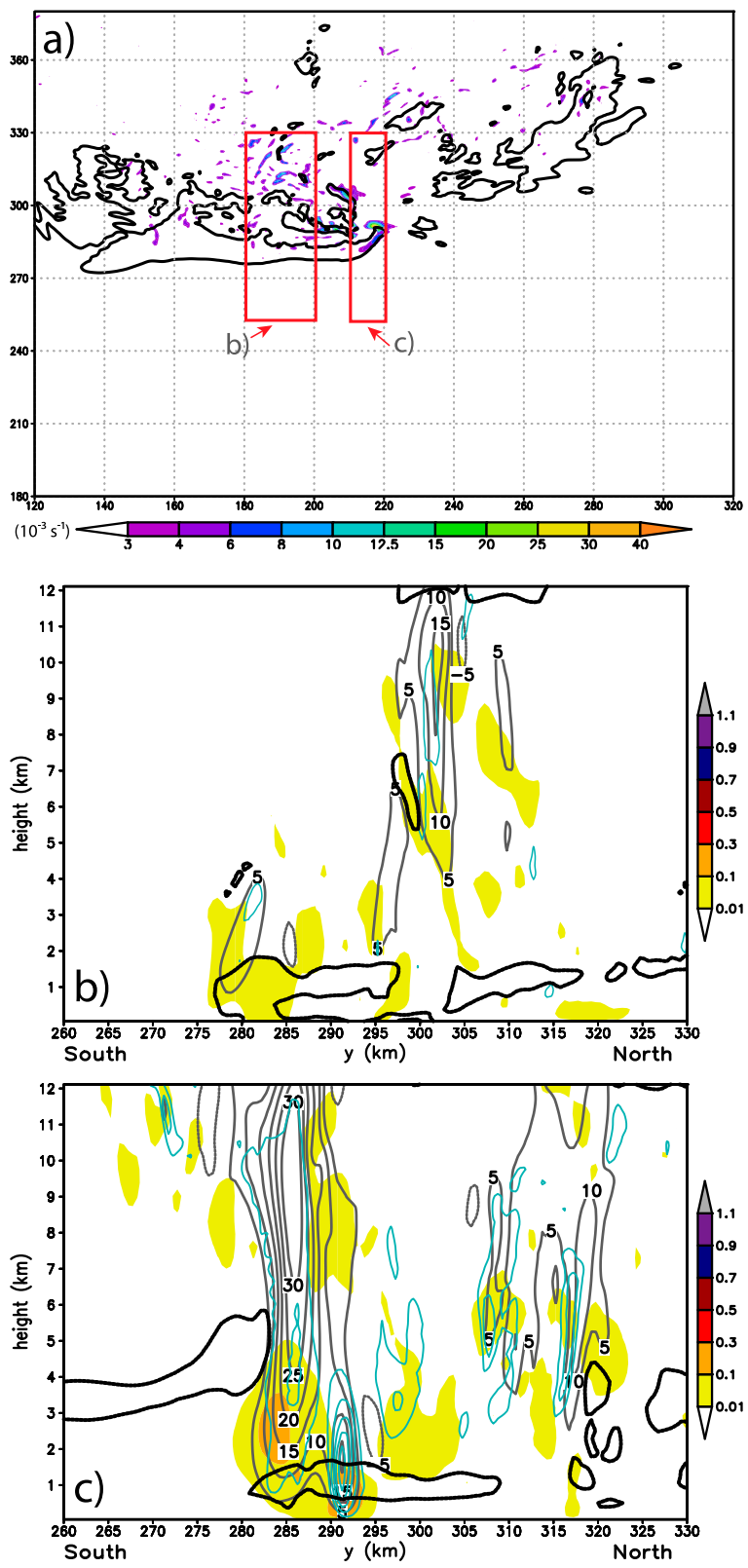

FIG. 17. (a) The 500-m vertical vorticity $\left(\times 10^{-3} \mathrm{~s}^{-1}\right)$ and surface perturbation potential temperature $\theta^{\prime}(-0.5 \mathrm{~K}$ contoured in black) for the CONTROL simulation $11 \mathrm{~h} 55 \mathrm{~min}$ into the model run. Red boxes in (a) indicate the east-west extend over which the (b),(c) north-south vertical cross sections were averaged. (b) Average north-south vertical cross section of dynamic NLD-VPPGF (shaded; $\mathrm{m} \mathrm{s}^{-2}$ ), perturbation potential temperature $\theta^{\prime}(-0.5 \mathrm{~K}$ contoured in black), vertical velocity $w$ (gray contours; contoured every $5 \mathrm{~m} \mathrm{~s}^{-1}$ ), and vertical vorticity (cyan contours, contoured every $5 \times 10^{-3} \mathrm{~s}^{-1}$ above $5 \times 10^{-3} \mathrm{~s}^{-1}$ ) for red box b in (a). (c) As in (b), but valid over the red box c in (a).

precipitation, and domain coverage of largest accumulation amounts (Figs. 18e,f, Table 2). Higher mean and maximum low-level updrafts are seen in the CONTROL_COR run compared to the LOW_SHEAR_COR (not shown), again following a similar pattern to the non-Coriolis simulations. While an in-depth analysis of perturbation pressure fields for the CONTROL_COR and LOW_SHEAR_COR is not presented in this manuscript, the similarities in the MCS morphology, vortex development, updraft strengths, accumulated precipitation, and how these characteristics scale with the $0-1-\mathrm{km}$ shear between the runs with and without Coriolis suggest that the mechanisms discussed above are not strongly sensitive to planetary rotation.

\section{Summary, discussion, and conclusions}

In summary, high-intensity, short-term extreme rainfall accumulations have been observed with concurrent and near-collocated mesoscale rotation. One such event that occurred in south-central Texas on 30 October 2015 served as motivation for several numerical simulations to determine the effects of intense $0-1-\mathrm{km}$ low-level shear and the resulting rotation on the accumulated precipitation. Various storm-scale aspects of the simulations were analyzed with a focus given to those related to precipitation intensity. Further, accelerations associated with the buoyant and dynamic components (i.e., linear and nonlinear) of the vertical perturbation pressure gradient force were calculated for each simulation to examine potential sources of vertical momentum not associated with thermodynamic buoyancy.

The resulting simulations produced similar MCSs with embedded supercells that all produced low-level vertical rotation, albeit at various strengths. The simulations with more intense $0-1-\mathrm{km}$ shear produced higher precipitation accumulations in the mean, point maximum, and domain coverage of the highest accumulations. Further, the strength and longevity of the low-level rotation increased with $0-1-\mathrm{km}$ shear magnitude. Similarly, the areal-mean and maximum low-level updrafts increased with increasing 0-1-km shear, as did the resulting low-level mass flux. Parcels in all of the simulations were regularly lifted out of the thermodynamically stable boundary layer, where cold pool development is limited in the CONTROL simulation compared to the other lower-shear runs. Accelerations from the NLD-VPPGF were found to dominate in the low levels over both linear dynamic and total buoyancy accelerations. These accelerations were found to increase in spatial extent, magnitude, and longevity as the $0-1-\mathrm{km}$ shear increased (i.e., from the CONTROL to the MED_SHEAR to the LOW_SHEAR runs), consistent with other studies. This is not surprising given that rotation around a vertical axis can contribute to the NLD-VPPGF [i.e., Eq. (4)], which in turn is highly correlated with large values of $0-1-\mathrm{km}$ shear. The higher NLD-VPPGF accelerations, which are an order of magnitude higher than the total buoyancy accelerations 

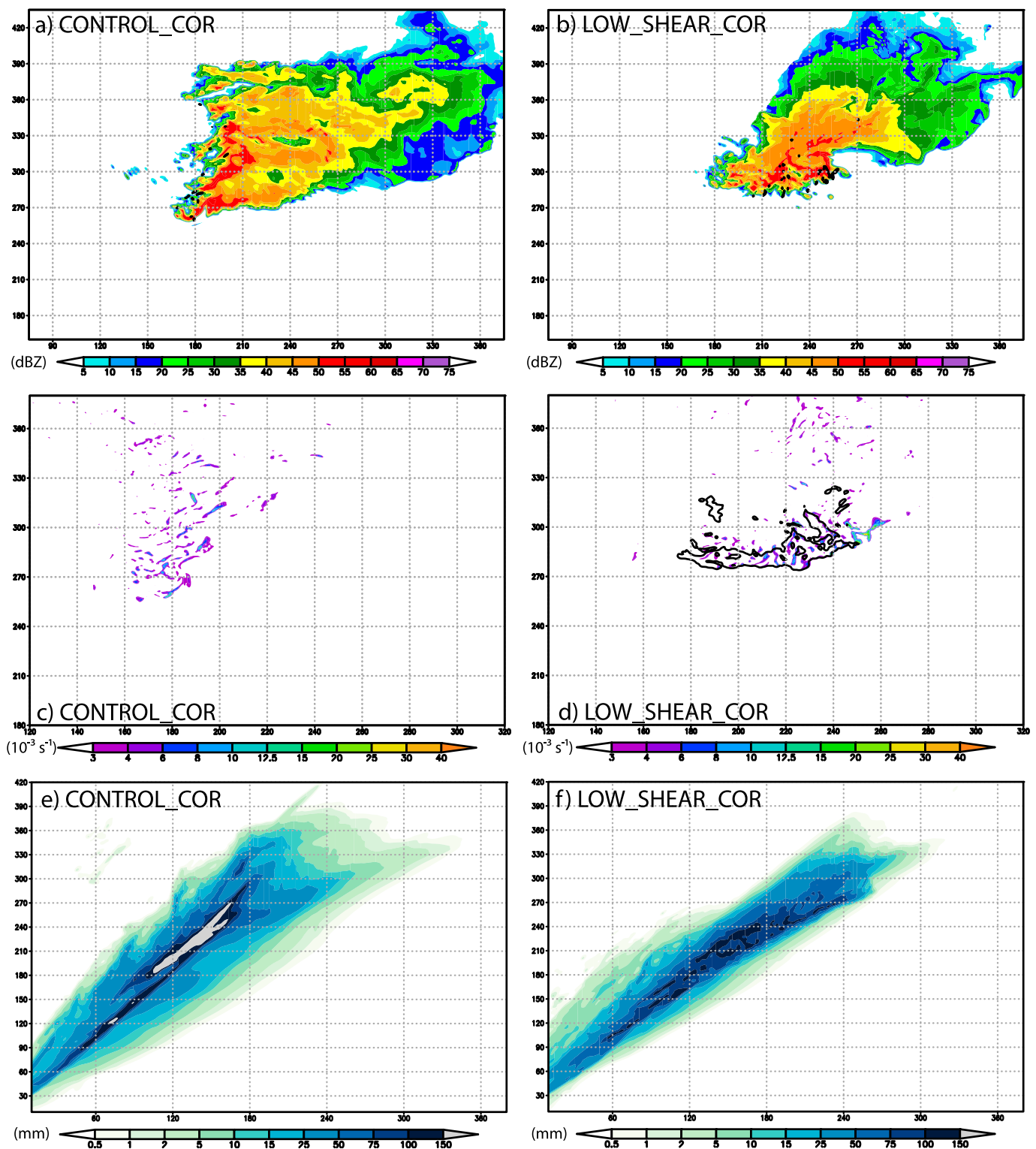

FIG. 18. (a),(b) Simulated 1-km radar reflectivity (shaded; every $5 \mathrm{~dB} Z$ from 5 to $70 \mathrm{~dB} Z$ ), surface perturbation potential temperature $\theta^{\prime}$ (contoured at -1.5 and $-2.5 \mathrm{~K}$ in dark purple and magenta, respectively), and contoured $1-\mathrm{km}$ vertical vorticity (black contours; starting at $10.0 \times 10^{-3} \mathrm{~s}^{-1}$ every $5.0 \times 10^{-3} \mathrm{~s}^{-1}$ ) valid $13 \mathrm{~h}$ into the simulation for the (a) CONTROL_COR and (b) LOW_SHEAR_COR simulations. (c),(d) The 1-km vertical vorticity $\left(\times 10^{-3} \mathrm{~s}^{-1}\right)$ for the (c) CONTROL_COR and (d) LOW_SHEAR_COR simulations valid at the same time as (a) and (b). (e),(f) Translated total accumulated precipitation (mm) in (e) CONTROL_COR and (f) LOW_SHEAR_COR simulations. Run-specific statistics are presented in Table 2.

(ACCB) at low levels, lead to lower, more intense updrafts in the simulations with stronger low-level shear.

The results of these simulations highlight the potential for mesocyclones or other meso- $\gamma$-scale vortices associated with intense $0-1-\mathrm{km}$ shear to enhance precipitation processes by enhancing low-level updrafts and, depending on the environmental thermodynamic profile, tapping into sources of moisture and instability that are otherwise difficult to ingest into the storm. The collocation of NLD-VPPGF associated acceleration with rotation (Figs. 15b,d,f and 17c), presence of low-level tracers at upper levels, persistent increase in low-level mass flux (Fig. 12), updraft strength (Fig. 10), and total accumulated precipitation (Fig. 9) in the CONTROL run versus the lower-shear simulations (i.e., MED_SHEAR and LOW_SHEAR) illustrate these 
points well. Previous literature has established that $0-1-\mathrm{km}$ shear is conducive for tornado development because it effectively lowers the base of the midlevel mesocyclone (e.g., Markowski et al. 2012; Markowski and Richardson 2014; Coffer and Parker 2015), which, in turn, makes it easier for the NLD-VPPGF associated with the rotation to lift negatively buoyant air (e.g., Nowotarski et al. 2011; Davenport and Parker 2015) from both the boundary layer and the cold pool. While the focus in this previous work was on the ability of the NLD-VPPGF to interact with baroclinically generated horizontal vorticity to lead to tornadogenesis, the experiments conducted in this study show that the same physical processes can simultaneously act to increase the ingredients needed for extreme rain rates. The enhancement of low-level updrafts, $w$ in Eq. (2), and the potential associated increase in the availability of moisture and CAPE, $q$ in Eq. (2), that otherwise would not be available to the system (e.g., Schumacher 2015b) leads to an increase in the instantaneous rain rate, $R$ in Eq. (2). Further, as suggested in the tornado literature, it is plausible that a positive feedback can occur between the initial rotation, enhancement of the updraft with the NLD-VPPGF, and increased rotation (e.g., Coffer and Parker 2015). Additionally, the longevity of MCSs and supercells, such as those simulated above, would also allow for potential feedbacks between precipitation process and rotation to occur, because of diabatic heating (e.g., Raymond and Jiang 1990; Weijenborg et al. 2017).

Note that the increase in $0-1-\mathrm{km}$ shear from the LOW_SHEAR to CONTROL run increases the strength of the storm-relative inflow and resultant moisture flux into the storm, which affects $q$ and $E$ in Eq. (2). The approximate increase in storm-relative inflow between each simulation is mainly related to the increase in wind speed through the $0-1-\mathrm{km}$ layer, since the layer moisture content and approximate storm motions are the same for each simulation. This increase in storm-relative moisture flux is quite substantial, near $30 \%$, between the LOW_SHEAR and CONTROL simulations at $500 \mathrm{~m}$ and is a compounding factor, along with the increase in low-level vertical motion, in the modeled increase in accumulated precipitation as $0-1-\mathrm{km}$ shear increases. However, a $\sim 60 \%$ increase in mean precipitation is seen from the LOW_SHEAR run to the CONTROL simulation, which cannot be explained by the increase in inflow alone. Further, the thermodynamically stable, moisture-laden air in the storm inflow still needs to be lifted out of the boundary layer, which is accomplished more effectively, compared to the lower-shear simulations, by the rotationally induced NLD-VPPGF in the CONTROL run that is also related to the magnitude of the $0-1-\mathrm{km}$ shear. In other words, the increase in low-level storm-relative inflow as the $0-1-\mathrm{km}$ shear increases is likely working in addition to the NLD-VPPGF to enhance precipitation accumulations in instances of intense $0-1-\mathrm{km}$ shear, though the influences of each individual process are difficult to isolate. Additionally, since only one thermodynamic profile was tested, thermodynamic sensitivities assuredly exist for the processes discussed in this manuscript.

The presence of intense 0-1-km shear (e.g., for various storm modes; Tuttle and Davis 2006; Morin and Parker 2011; Markowski and Richardson 2014) and the development of isolated rotation or embedded rotation in MCSs/MCVs (e.g., Morales et al. 2015), tropical cyclone rainbands (e.g., Edwards et al. 2012; Wang et al. 2015), and supercells, in theory, means that precipitation enhancement, as described in the manuscript, could be seen in many different storm morphologies. This mechanism can serve to explain why supercells are often associated with intense rain rates despite low precipitation efficiency (e.g., Smith et al. 2001; Duda and Gallus 2010; Hitchens and Brooks 2013). The enhancement of vertical momentum and, thus, precipitation by these mechanisms does not, in principle, preclude the formation of a tornado, since the same mechanisms (i.e., intense, dynamically induced updrafts near the surface) are favorable for tornadogenesis (e.g., Markowski and Richardson 2014). Additionally, the potential for simultaneous enhancement of both rainfall intensity and tornado potential provides a potential explanation for the observed frequency, around 80 events per year between 2003 and 2015 (Nielsen et al. 2017), of concurrent, collocated tornado and flash flood events (TORFFs) that occur in isolated supercells, organized MCSs, and tropical cyclones (Nielsen et al. 2015) without any clear dependence on storm motion (Bunkers and Doswell 2016; Nielsen et al. 2016a).

In conclusion, precipitation systems in intense $0-1-\mathrm{km}$ shear that develop mesoscale rotation can aid in producing extreme precipitation by enhancing the magnitude of lowlevel updrafts through accelerations associated with rotationally induced nonlinear dynamic vertical perturbation pressure gradient forces. The resulting increase in low-level vertical motion can further serve to enhance precipitation, depending on the environmental conditions, by ingesting otherwise negatively buoyant parcels that still contain moisture and CAPE. These precipitation enhancements could be more pronounced in situations where thermodynamic buoyancy is limited and moisture is abundant. Ongoing work is examining rain gauge and gridded precipitation data to determine the propensity for extreme short-term rainfall accumulations (i.e., $>75 \mathrm{~mm} \mathrm{~h}^{-1}$ ) to be associated with near concurrent, collocated mesoscale 
rotation outside of the motivating case presented in this research.

Acknowledgments. The authors thank Gregory Herman, Stacey Hitchcock, Keith Sherburn, Leah Grant, John Peters, and Lance Wood for their helpful comments on this work. Furthermore, the authors thank Brice Coffer and Matthew Parker for the pressure perturbation decomposition code and useful analysis suggestions. Helpful commentary on this paper was provided by Matthew Bunkers and two anonymous reviewers. High-performance computing resources from Cheyenne (https://doi.org/10.5065/D6RX99HX) and Yellowstone (ark:/85065/d7wd3xhc) were provided by the National Center for Atmospheric Research (NCAR), which is sponsored by the National Science Foundation. METAR data were provided by the Iowa Environmental Mesonet. The Rapid Refresh (RAP) analysis was provided by the National Oceanic and Atmospheric Administration's National Centers for Environmental Prediction. This research was supported by National Science Foundation Grant AGS-1359727, NOAA Grants NA15OAR4590233 and NA16OAR4590215, and a National Science Foundation Graduate Research Fellowship Grant DGE-1321845, Amendment 3.

\section{REFERENCES}

Adlerman, E. J., K. K. Droegemeier, and R. Davies-Jones, 1999: A numerical simulation of cyclic mesocyclogenesis. J. Atmos. Sci., 56, 2045-2069, https://doi.org/10.1175/1520-0469(1999) $056<2045$ :ANSOCM $>2.0$. CO;2.

Ashley, S. T., and W. S. Ashley, 2008: Flood fatalities in the United States. J. Appl. Meteor. Climatol., 47, 805-818, https://doi.org/ 10.1175/2007JAMC1611.1.

Atkins, N. T., and M. St. Laurent, 2009: Bow echo mesovortices. Part I: Processes that influence their damaging potential. Mon. Wea. Rev., 137, 1497-1513, https://doi.org/10.1175/2008MWR2649.1.

Baker, A. K., M. D. Parker, and M. D. Eastin, 2009: Environmental ingredients for supercells and tornadoes within Hurricane Ivan. Wea. Forecasting, 24, 223-244, https://doi.org/10.1175/ 2008WAF2222146.1.

Beatty, K., E. Rasmussen, and J. Straka, 2008: The supercell spectrum. Part I: A review of research related to supercell precipitation morphology. Electron. J. Severe Storms Meteor., 3 (4), http://www.ejssm.org/ojs/index.php/ejssm/article/viewArticle/44.

Benjamin, S. G., and Coauthors, 2016: A North American hourly assimilation and model forecast cycle: The Rapid Refresh. Mon. Wea. Rev., 144, 1669-1694, https://doi.org/10.1175/ MWR-D-15-0242.1.

Bluestein, H. B., and M. H. Jain, 1985: Formation of mesoscale lines of precipitation: Severe squall lines in Oklahoma during the spring. J. Atmos. Sci., 42, 1711-1732, https://doi.org/ 10.1175/1520-0469(1985)042<1711:FOMLOP > 2.0.CO;2.

Bonner, W. D., 1968: Climatology of the low level jet. Mon. Wea. Rev., 96, 833-850, https://doi.org/10.1175/1520-0493(1968) 096<0833:COTLLJ $>2.0$.CO;2.
Bosart, L. F., and F. Sanders, 1981: The Johnstown flood of July 1977: A long-lived convective system. J. Atmos. Sci., 38, 1616-1642, https:// doi.org/10.1175/1520-0469(1981)038<1616:TJFOJA >2.0.CO;2.

Browning, K. A., 1977: The structure and mechanism of hailstorms. Hail: A Review of Hail Science and Hail Suppression, Meteor. Monogr., No. 38, Amer. Meteor. Soc., 1-43.

Bryan, G. H., and J. M. Fritsch, 2002: A benchmark simulation for moist nonhydrostatic numerical models. Mon. Wea. Rev., 130, 2917-2928, https://doi.org/10.1175/1520-0493(2002)130<2917: ABSFMN $>2.0 . \mathrm{CO} ; 2$.

Bunkers, M. J., and C. A. Doswell III, 2016: Comments on "Double impact: When both tornadoes and flash floods threaten the same place at the same time." Wea. Forecasting, 31, 17151721, https://doi.org/10.1175/WAF-D-16-0116.1.

B. A. Klimowski, J. W. Zeitler, R. L. Thompson, and M. L. Weisman, 2000: Predicting supercell motion using a new hodograph technique. Wea. Forecasting, 15, 61-79, https://doi.org/ 10.1175/1520-0434(2000)015<0061:PSMUAN>2.0.CO;2.

Chagnon, J. M., and S. L. Gray, 2009: Horizontal potential vorticity dipoles on the convective storm scale. Quart. J. Roy. Meteor. Soc., 135, 1392-1408, https://doi.org/10.1002/qj.468.

Chappell, C. F., 1986: Quasi-stationary convective events. Mesoscale Meteorology and Forecasting, P. S. Ray, Ed., Amer. Meteor. Soc., 289-309.

Clark, A. J., J. Gao, P. T. Marsh, T. Smith, J. S. Kain, J. Correia Jr., M. Xue, and F. Kong, 2013: Tornado pathlength forecasts from 2010 to 2011 using ensemble updraft helicity. Wea. Forecasting, 28, 387-407, https://doi.org/10.1175/WAF-D-12-00038.1.

Coffer, B. E., and M. D. Parker, 2015: Impacts of increasing lowlevel shear on supercells during the early evening transition. Mon. Wea. Rev., 143, 1945-1969, https://doi.org/10.1175/ MWR-D-14-00328.1.

Craven, J. P., H. E. Brooks, and J. A. Hart, 2004: Baseline climatology of sounding derived parameters associated with deep, moist convection. Natl. Wea. Dig., 28 (1), 13-24, http:// nwafiles.nwas.org/digest/papers/2004/Vol28/Pg13-Craven.pdf.

Dalrymple, T., 1937: Major Texas floods of 1935. U.S. Geological Survey Water Supply Paper 796-G, 287 pp.

Davenport, C. E., and M. D. Parker, 2015: Observations of the 9 June 2009 dissipating supercell from VORTEX2. Wea. Forecasting, 30, 368-388, https://doi.org/10.1175/ WAF-D-14-00087.1.

Doswell, C. A., III, 1994: Flash flood producing convective storms: Current understanding and research. Proc. U.S.-Spain Joint Workshop on Natural Hazards, Barcelona, Spain, National Science Foundation, 97-107.

_ H. E. Brooks, and R. A. Maddox, 1996: Flash flood forecasting: An ingredients-based methodology. Wea. Forecasting, 11, 560-581, https://doi.org/10.1175/1520-0434(1996)011<0560: FFFAIB $>2.0 . \mathrm{CO} ; 2$.

Duda, J. D., and W. A. Gallus Jr., 2010: Spring and summer midwestern severe weather reports in supercells compared to other morphologies. Wea. Forecasting, 25, 190-206, https:// doi.org/10.1175/2009WAF2222338.1.

Durran, D. R., and J. B. Klemp, 1983: A compressible model for the simulation of moist mountain waves. Mon. Wea. Rev., 111, 2341-2361, https://doi.org/10.1175/1520-0493(1983)111<2341: ACMFTS $>2.0 . \mathrm{CO} ; 2$.

Edwards, R., A. R. Dean, R. L. Thompson, and B. T. Smith, 2012: Convective modes for significant severe thunderstorms in the contiguous United States. Part III: Tropical cyclone tornadoes. Wea. Forecasting, 27, 1507-1519, https://doi.org/ 10.1175/WAF-D-11-00117.1. 
Foote, G., and J. Fankhauser, 1973: Airflow and moisture budget beneath a northeast Colorado hailstorm. J. Appl. Meteor., 12, 1330-1353, https://doi.org/10.1175/1520-0450(1973)012<1330: AAMBBA $>2.0 . \mathrm{CO} ; 2$.

Fritsch, J. M., and R. Carbone, 2004: Improving quantitative precipitation forecasts in the warm season: A USWRP research and development strategy. Bull. Amer. Meteor. Soc., 85, 955965, https://doi.org/10.1175/BAMS-85-7-955.

— , R. Kane, and C. Chelius, 1986: The contribution of mesoscale convective weather systems to the warm-season precipitation in the United States. J. Climate Appl. Meteor., 25, 1333-1345, https:// doi.org/10.1175/1520-0450(1986)025<1333:TCOMCW>2.0.CO;2.

_ J. D. Murphy, and J. S. Kain, 1994: Warm core vortex amplification over land. J. Atmos. Sci., 51, 1780-1807, https://doi.org/ 10.1175/1520-0469(1994)051<1780:WCVAOL $>2.0 . C O ; 2$.

Haynes, P., and M. McIntyre, 1987: On the evolution of vorticity and potential vorticity in the presence of diabatic heating and frictional or other forces. J. Atmos. Sci., 44, 828-841, https://doi. org/10.1175/1520-0469(1987)044<0828:OTEOVA >2.0.CO;2.

Herman, G. R., and R. S. Schumacher, 2016: Extreme precipitation in models: An evaluation. Wea. Forecasting, 31, 1853-1879, https://doi.org/10.1175/WAF-D-16-0093.1.

$\longrightarrow$, and — 2018: Money doesn't grow on trees, but forecasts do: Forecasting extreme precipitation with random forests. Mon. Wea. Rev., 146, 1571-1600, https://doi.org/10.1175/ MWR-D-17-0250.1.

Hertenstein, R. F., and W. H. Schubert, 1991: Potential vorticity anomalies associated with squall lines. Mon. Wea. Rev., 119, 1663-1672, https://doi.org/10.1175/1520-0493(1991)119<1663: PVAAWS $>2.0 . \mathrm{CO} ; 2$.

Hitchens, N. M., and H. E. Brooks, 2013: Preliminary investigation of the contribution of supercell thunderstorms to the climatology of heavy and extreme precipitation in the United States. Atmos. Res., 123, 206-210, https://doi.org/10.1016/j.atmosres.2012.06.023.

James, E. P., and R. H. Johnson, 2010: Patterns of precipitation and mesolow evolution in midlatitude mesoscale convective vortices. Mon. Wea. Rev., 138, 909-931, https://doi.org/10.1175/ 2009MWR3076.1.

Klemp, J. B., 1987: Dynamics of tornadic thunderstorms. Annu. Rev. Fluid Mech., 19, 369-402, https://doi.org/10.1146/ annurev.fl.19.010187.002101.

— W. C. Skamarock, and J. Dudhia, 2007: Conservative splitexplicit time integration methods for the compressible nonhydrostatic equations. Mon. Wea. Rev., 135, 2897-2913, https://doi.org/10.1175/MWR3440.1.

LCRA, 2017: Lower Colorado River Authority: Historical data by gauge. LCRA, accessed 28 September 2017, https://hydromet. lcra.org/HistoricalData.

Loftus, A. M., D. B. Weber, and C. A. Doswell III, 2008: Parameterized mesoscale forcing mechanisms for initiating numerically simulated isolated multicellular convection. Mon. Wea. Rev., 136, 2408-2421, https://doi.org/10.1175/2007MWR2133.1.

Markowski, P., and Y. Richardson, 2010: Mesoscale Meteorology in Midlatitudes. John Wiley and Sons, 430 pp.

— shear and cold pools on tornadogenesis: Insights from idealized simulations. J. Atmos. Sci., 71, 243-275, https://doi.org/ 10.1175/JAS-D-13-0159.1.

_- , and Coauthors, 2012: The pretornadic phase of the Goshen County, Wyoming, supercell of 5 June 2009 intercepted by VORTEX2. Part II: Intensification of low-level rotation. Mon. Wea. Rev., 140, 2916-2938, https://doi.org/10.1175/ MWR-D-11-00337.1.
Marwitz, J. D., 1972: Precipitation efficiency of thunderstorms on the high plains. J. Rech. Atmos., 6, 367-370.

McCaul, E. W., Jr., and M. L. Weisman, 2001: The sensitivity of simulated supercell structure and intensity to variations in the shapes of environmental buoyancy and shear profiles. Mon. Wea. Rev., 129, 664-687, https://doi.org/10.1175/1520-0493(2001)129<0664: TSOSSS $>2.0 . \mathrm{CO} ; 2$.

Moller, A. R., C. A. Doswell III, M. P. Foster, and G. R. Woodall, 1994: The operational recognition of supercell thunderstorm environments and storm structures. Wea. Forecasting, 9, 327-347, https://doi.org/10.1175/1520-0434(1994)009<0327: TOROST $>2.0 . \mathrm{CO} ; 2$.

Moore, J. T., F. H. Glass, C. E. Graves, S. M. Rochette, and M. J. Singer, 2003: The environment of warm-season elevated thunderstorms associated with heavy rainfall over the central United States. Wea. Forecasting, 18, 861-878, https://doi.org/ 10.1175/1520-0434(2003)018<0861:TEOWET >2.0.CO;2.

Morales, A., R. S. Schumacher, and S. M. Kreidenweis, 2015: Mesoscale vortex development during extreme precipitation: Colorado, September 2013. Mon. Wea. Rev., 143, 4943-4962, https://doi.org/10.1175/MWR-D-15-0086.1.

Morin, M. J., and M. D. Parker, 2011: A numerical investigation of supercells in landfalling tropical cyclones. Geophys. Res. Lett., 38, L10801, https://doi.org/10.1029/2011GL047448.

Morrison, H. G., G. Thompson, and V. Tatarskii, 2009: Impact of cloud microphysics on the development of trailing stratiform precipitation in a simulated squall line: Comparison of oneand two-moment schemes. Mon. Wea. Rev., 137, 991-1007, https://doi.org/10.1175/2008MWR2556.1.

NCEI, 2017: Storm events database. NOAA, accessed 26 November 2017, http://www.ncdc.noaa.gov/stormevents/.

Nielsen, E. R., and R. S. Schumacher, 2016: Using convectionallowing ensembles to understand the predictability of an extreme rainfall event. Mon. Wea. Rev., 144, 3651-3676, https://doi.org/10.1175/MWR-D-16-0083.1.

_ G. R. Herman, R. C. Tournay, J. M. Peters, and R. S. Schumacher, 2015: Double impact: When both tornadoes and flash floods threaten the same place at the same time. Wea. Forecasting, 30 , 1673-1693, https://doi.org/10.1175/WAF-D-15-0084.1.

, and _ 2016a: Reply to "Comments on 'Double impact: When both tornadoes and flash floods threaten the same place at the same time."'Wea. Forecasting, 31, 1723-1727, https://doi.org/10.1175/WAF-D-16-0151.1.

- R. S. Schumacher, and A. M. Keclik, 2016b: The effect of the Balcones Escarpment on three cases of extreme precipitation in central Texas. Mon. Wea. Rev., 144,119-138, https://doi.org/ 10.1175/MWR-D-15-0156.1.

_, G. R. Herman, and R. S. Schumacher, 2017: An updated U.S. geographic distribution of concurrent, collocated tornado and flash flood events and a look at those observed during the first year of VORTEX-SE. Special Symp. on Severe Local Storms, Seattle, WA, Amer. Meteor. Soc., 923, https://ams.confex. com/ams/97Annual/webprogram/Paper305034.html.

Novak, D. R., C. Bailey, K. Brill, M. Eckert, D. Petersen, R. Rausch, and M. Schichtel, 2011: Human improvement to numerical weather prediction at the Hydrometeorological Prediction Center. 24th Conf. on Weather and Forecasting-20th Conf. on Numerical Weather Prediction, Seattle, WA, Amer. Meteor Soc., 440, https://ams.confex.com/ams/91Annual/webprogram/ Paper181989.html.

Nowotarski, C. J., P. M. Markowski, and Y. P. Richardson, 2011: The characteristics of numerically simulated supercell storms situated over statically stable boundary layers. Mon 
Wea. Rev., 139, 3139-3162, https://doi.org/10.1175/MWR-D10-05087.1.

Parker, M. D., and R. H. Johnson, 2000: Organizational modes of midlatitude mesoscale convective systems. Mon. Wea. Rev., 128, 3413-3436, https://doi.org/10.1175/1520-0493(2001) 129<3413:OMOMMC $>2.0 . \mathrm{CO} ; 2$.

— , and — 2004: Structures and dynamics of quasi-2D mesoscale convective systems. J. Atmos. Sci., 61, 545-567, https://doi.org/ 10.1175/1520-0469(2004)061<0545:SADOQM >2.0.CO;2.

Peters, J. M., E. R. Nielsen, M. D. Parker, S. M. Hitchcock, and R. S. Schumacher, 2017: The impact of low-level moisture errors on model forecasts of an MCS observed during PECAN. Mon. Wea. Rev., 145, 3599-3624, https://doi.org/ 10.1175/MWR-D-16-0296.1.

Raymond, D., and H. Jiang, 1990: A theory for long-lived mesoscale convective systems. J. Atmos. Sci., 47, 3067-3077, https://doi.org/ 10.1175/1520-0469(1990)047<3067:ATFLLM>2.0.CO;2.

Rotunno, R., and J. B. Klemp, 1982: The influence of the shearinduced pressure gradient on thunderstorm motion. Mon Wea. Rev., 110, 136-151, https://doi.org/10.1175/1520-0493(1982) 110<0136:TIOTSI $>2.0 . \mathrm{CO} ; 2$.

Schumacher, R. S., 2009: Mechanisms for quasi-stationary behavior in simulated heavy-rain-producing convective systems. J. Atmos. Sci., 66, 1543-1568, https://doi.org/10.1175/ 2008JAS2856.1.

_ $2015 \mathrm{a}$ : Resolution dependence of initiation and upscale growth of deep convection in convection-allowing forecasts of the 31 May-1 June 2013 supercell and MCS. Mon. Wea. Rev., 143, 4331-4354, https://doi.org/10.1175/MWR-D-15-0179.1. ,2015b: Sensitivity of precipitation accumulation in elevated convective systems to small changes in low-level moisture. J. Atmos. Sci., 72, 2507-2524, https://doi.org/10.1175/JAS-D-14-0389.1.

_ , and R. H. Johnson, 2005: Organization and environmental properties of extreme-rain-producing mesoscale convective systems. Mon. Wea. Rev., 133, 961-976, https://doi.org/10.1175/ MWR2899.1.

— , and - 2006: Characteristics of U.S. extreme rain events during 1999-2003. Wea. Forecasting, 21, 69-85, https://doi.org/ 10.1175/WAF900.1.

— convective systems associated with midlevel cyclonic circulations. Wea. Forecasting, 24, 555-574, https://doi.org/10.1175/ 2008WAF2222173.1.

_- A. J. Clark, M. Xue, and F. Kong, 2013: Factors influencing the development and maintenance of nocturnal heavy-rainproducing convective systems in a storm-scale ensemble Mon. Wea. Rev., 141, 2778-2801, https://doi.org/10.1175/ MWR-D-12-00239.1.

Skamarock, W. C., and J. B. Klemp, 2008: A time-split nonhydrostatic atmospheric model for weather research and forecasting applications. J. Comput. Phys., 227, 3465-3485, https://doi.org/10.1016/j.jcp.2007.01.037.

, and Coauthors, 2008: A description of the Advanced Research WRF version 3. NCAR Tech. Note NCAR/TN-475+STR, 113 pp., https://doi.org/10.5065/D68S4MVH.
Smith, J. A., M. L. Baeck, Y. Zhang, and C. A. Doswell III, 2001: Extreme rainfall and flooding from supercell thunderstorms. J. Hydrometeor., 2, 469-489, https://doi.org/10.1175/1525-7541 (2001)002<0469:ERAFFS $>2.0$. CO;2.

Stevenson, S. N., and R. S. Schumacher, 2014: A 10-year survey of extreme rainfall events in the central and eastern United States using gridded multisensor precipitation analyses. Mon. Wea. Rev., 142, 3147-3162, https://doi.org/10.1175/ MWR-D-13-00345.1.

Trapp, R. J., and M. L. Weisman, 2003: Low-level mesovortices within squall lines and bow echoes. Part II: Their genesis and implications. Mon. Wea. Rev., 131, 2804-2823, https://doi.org/ 10.1175/1520-0493(2003)131<2804:LMWSLA > 2.0.CO;2.

Trier, S. B., C. A. Davis, and J. D. Tuttle, 2000a: Long-lived mesoconvective vortices and their environment. Part I: Observations from the central United States during the 1998 warm season. Mon. Wea. Rev., 128, 3376-3395, https://doi.org/ 10.1175/1520-0493(2000)128<3376:LLMVAT>2.0.CO;2.

,,$\ldots$, and W. C. Skamarock, 2000b: Long-lived mesoconvective vortices and their environment. Part II: Induced thermodynamic destabilization in idealized simulations. Mon. Wea. Rev., 128, 3396-3412, https://doi.org/10.1175/1520-0493(2000)128<3396: LLMVAT $>2.0 . \mathrm{CO} ; 2$.

Tuttle, J. D., and C. A. Davis, 2006: Corridors of warm season precipitation in the central United States. Mon. Wea. Rev., 134, 2297-2317, https://doi.org/10.1175/MWR3188.1.

Wang, C.-C., H.-C. Kuo, R. H. Johnson, C.-Y. Lee, S.-Y. Huang, and Y.-H. Chen, 2015: A numerical study of convection in rainbands of Typhoon Morakot (2009) with extreme rainfall: Roles of pressure perturbations with low-level wind maxima. Atmos. Chem. Phys., 15, 11 097-11 115, https://doi.org/ 10.5194/acp-15-11097-2015.

Weijenborg, C., P. Friederichs, and A. Hense, 2015: Organisation of potential vorticity on the mesoscale during deep moist convection. Tellus, 67A, 25705, https://doi.org/10.3402/tellusa.v67.25705.

_ J. Chagnon, P. Friederichs, S. Gray, and A. Hense, 2017: Coherent evolution of potential vorticity anomalies associated with deep moist convection. Quart. J. Roy. Meteor. Soc., 143, 1254-1267, https://doi.org/10.1002/qj.3000

Weisman, M. L., and J. B. Klemp, 1984: The structure and classification of numerically simulated convective storms in directionally varying wind shears. Mon. Wea. Rev., 112, 2479-2498, https://doi.org/10.1175/1520-0493(1984)112<2479: TSACON $>2.0 . \mathrm{CO} ; 2$.

- and R. J. Trapp, 2003: Low-level mesovortices within squall lines and bow echoes. Part I: Overview and dependence on environmental shear. Mon. Wea. Rev., 131, 2779-2803, https://doi.org/ 10.1175/1520-0493(2003)131<2779:LMWSLA > 2.0.CO;2.

Wilhelmson, R., and Y. Ogura, 1972: The pressure perturbation and the numerical modeling of a cloud. J. Atmos. Sci., 29, 1295-1307, https://doi.org/10.1175/1520-0469(1972)029<1295: TPPATN $>2.0 . \mathrm{CO} ; 2$.

Zhang, J., and Coauthors, 2016: Multi-Radar Multi-Sensor (MRMS) quantitative precipitation estimation: Initial operating capabilities. Bull. Amer. Meteor. Soc., 97, 621-638, https://doi.org/ 10.1175/BAMS-D-14-00174.1. 